\title{
NUMERICAL STUDY OF UNCOUPLED AND COUPLED TLP MODELS
}

\author{
Jianwei Wang* \\ Yuankui Luo \\ Rui Guo \\ Wei Cai \\ Jingyi Zhao \\ Xiongshuai Luo \\ Yanshan University, Qinhuangdao, China \\ *Corresponding author: wangjianwei@ysu.edu.cn (J. Wang)
}

\begin{abstract}
Abstract: In this paper, two analysis models for tension leg platform (TLP) are proposed based on different simulation methods of the tendons for studying the TLP motion responses in waves. In the uncoupled analysis model, the tendon is simplified as a spring, and the restoring forces matrix is derived with the consideration of the influence of the coupled effect of horizontal offset and vertical setdown of the platform. In the coupled model, the axial and transverse vibration's coupled effect has been considered for the establishment of the vibration equations for the tendons, and the finite difference method is used to solve the vibration equations. The time-domain coupled motion model of the platform and the mooring system is established based on the interaction forces between the tendons and the platform. The coupled and uncoupled TLP models are compared and analysed to determine their applicability. Compared with the uncoupled TLP model, the coupled TLP model has greater accuracy and a wider application range, and the effects of second-order wave force on the platform responses, horizontal offset, and vertical subsidence are analysed.
\end{abstract}

Keywords: Coupled TLP model; Uncoupled TLP model; Hydynamics; Second-order wave force; Offset and setdown

\section{INTRODUCTION}

The tension leg platform (TLP) models can be calculated using the frequency domain method or the time domain method. The International Conference on Ship Structure (ISSC) and the American Petroleum Institute (API) [1] divided the time domain model for TLP into two categories: the uncoupled TLP model and the coupled TLP model.

At first, Paulling [2,3] proposed two uncoupled TLP models which were used to calculate the motions of the platform with three degrees of freedom and six degrees of freedom, respectively. The Morrison equation is used to calculate the wave forces. The wave forces act on the platform, and tendons need to be predicted accurately to design a robust mooring system.
A method for predicting the motions of and forces on the platform in regular or random waves was developed based on the linearised hydrodynamic method. The experimental results were in good agreement with the predicted results. Angelides [4] proposed a similar computational model that simplifies tendons as linear springs. Malaeb [5] introduced a set of 6x6 stiffness matrices for the calculation of TLP, in which the tendons were also simplified as linear springs. Although Malaeb's stiffness matrix representation method was proposed nearly 30 years ago, it is still widely used for analysing the uncoupled TLP model. Chandrasekaran [6] calculated the dynamic responses of the triangular TLP in the random waves based on the uncoupled model in which the influence of diffraction effect and second-order wave force was ignored. Kawanishi [7] studied 
the responses between TLP and pile foundation under the seismic excitation based on the uncoupled model. The results showed that the uplift capacity of the pile foundation must be more than twice the initial net buoyancy. Zhai [8] used the uncoupled model to calculate hydrodynamic parameters in the frequency domain, such as the transfer function, added mass, and damping coefficient. The motion responses of the platform under the action of wind and waves were calculated using the non-linear time-domain analytical method. According to the time series of the motion responses, the influence of Jonswap spectrum's parameters (including wave height and peak factors) on the heave responses of the platform was analysed.

Chandrasekaran [9] compared the triangle and four columns of TLP and analysed them using the Mathieu equation. The analysis results showed that the platform motion becomes unstable as the tendon length increases but increasing the pretension of the tendon can improve the stability of the platform. Low [10] linearised the non-linear stiffness matrix and analysed the motion responses of the traditional TLP. He also achieved the coupling between degrees of freedom through linearization techniques. Senjanović [11] proposed a consistent formulation of the stiffness matrix, derived from the general solution established for hydroelastic analysis of ship structures, as a specific case. The stiffness was compared to the known ones, and discrepancies were analysed and discussed.

The coupled TLP model is achieved by replacing the linear spring system with tethers and their material properties and mass. Because the distributed mass of tethers and the influence of resistance and additional mass are considered in the coupled TLP model, the accuracy of the calculated results will be improved significantly. At the same time, these factors may cause the transverse and axial excitation of tethers, affecting the platform's movements. In addition, it should be noted that considering the mass of tethers will also affect the static deformation of the structure due to its own gravity [12]. Jameel [13] studied the TLP motion and its tether tension under extreme sea conditions. 2 The wave-surface elevation is calculated by using the theory of small amplitude waves. Jain [14] considered the coupling effect between the six degrees of freedom of the platform and analysed the nonlinearity of the platform's motion caused by the change of tether tension and wave dragging force.

Faltinsen [15] aimed to provide a physical understanding through simplified mathematical models and introduced linearwave induced motions, loads on floating structures, numerical methods for ascertaining wave-induced motions and loads, viscous wave loads and damping, stationkeeping, and water impact and entry. Chatterjee [16] considered the elasticity of the platform decks and columns when calculating the coupled motion of TLP. Choi [17] conducted experiments and numerical analysis on the coupling characteristics of TLP and flexible semisubmersible floating structures. The free damping tests of TLP and semi-submerged platforms showed that they had complex coupling characteristics and multiple natural modal frequency components. Ran [18] used WINPOST FEA software to model tethers and developed a coupled Spar model. At the same time, wave forces were evaluated using the WINPOST\&WINTCOL project. Mazarakos [19] introduced a coupled analysis method for the mean second-order loads of a floating structure, which was suitable for offshore wind energy sources exploitation.

The time domain numerical calculation methods for TLP under the combined action of waves and currents often involve the problems of calculating the hydrodynamic performance of the platform, the non-linear vibration problem of the tethers, and the coupled motions of the platform and the tethers. For the research methods of the mooring system, most scholars have simplified the tethers into a massless spring structure. The coupled mathematical model in this paper fully considers the coupled effect of the axial and transverse vibration of the tethers. For the research methods of wave forces on the TLP, most scholars have adopted the Morison formula to direct estimation. In this paper, a combination of the potential flow theory and Morison formula is used to calculate the wave forces, which can effectively consider the effect of the diffraction potential on TLP. Based on the previous studies on TLP from the different scholars, the two TLP analytical models are further improved through the whole work. In the uncoupled TLP model, the coupled effects of horizontal offset and vertical setdown of the platform are considered in the restoring force coefficient matrix. In the coupled model, the coupled effects of axial and transverse vibrations of the tethers are considered. The two models are compared and analysed, and the applicable conditions are given.

\section{MATHEMATICAL MODEL OF THE UNCOUPLED TLP}

When TLP moves in real sea conditions, it will show wavefrequency movement and present horizontal offset and vertical setdown under the action of external loads such as wind load, current load, and mean second-order drift force. Moreover, because the riser system of TLP is arranged in a cluster manner, the horizontal deviation of the platform may cause interference between the risers of serial arrangement. Therefore, it is necessary to study the influence of horizontal deviation and vertical subsidence on TLP. Fig. 1 shows the structure diagram of the uncoupled TLP model in this case. The model simplifies the tether into a linear spring structure and is used to analyse the motion responses of TLP when considering the coupled effects of horizontal offset and vertical setdown of the platform.

In this paper, the platform is regarded as a rigid body, and the movement of the tethers is regarded as the elastic movement of the spring along with the movement of the platform. Therefore, the uncoupled TLP motion equation under the influence of tethers is given by:

$$
\left(M_{i j}+\mu_{i j}\right) \ddot{x}_{j}(t)+K_{i j} \dot{x}_{j}(t)+\bar{C}_{i j} x_{j}(t)=F_{i W}(t)+F_{i D}(t)
$$

where $x_{j}(j=1,2 \ldots, 6)$ is the movement of the TLP; $M_{i j}(i, j=1,2 \ldots, 6)$ is the sum of the mass of the floating structure and the vertical pipe considering the pretension force; $\mu_{i j}$ is the added mass of floating structure; $K_{i j}$ is the damping matrix; $\bar{C}_{i j}$ is the coefficient of restoring force matrix considering the effect of tethers; $F_{i W}$ is the sum forces of Froude-Krylov force 
and diffraction force; $F_{i D}$ is the drag force. The methods of the hydrodynamic coefficients of the platform can be found in Appendix A.

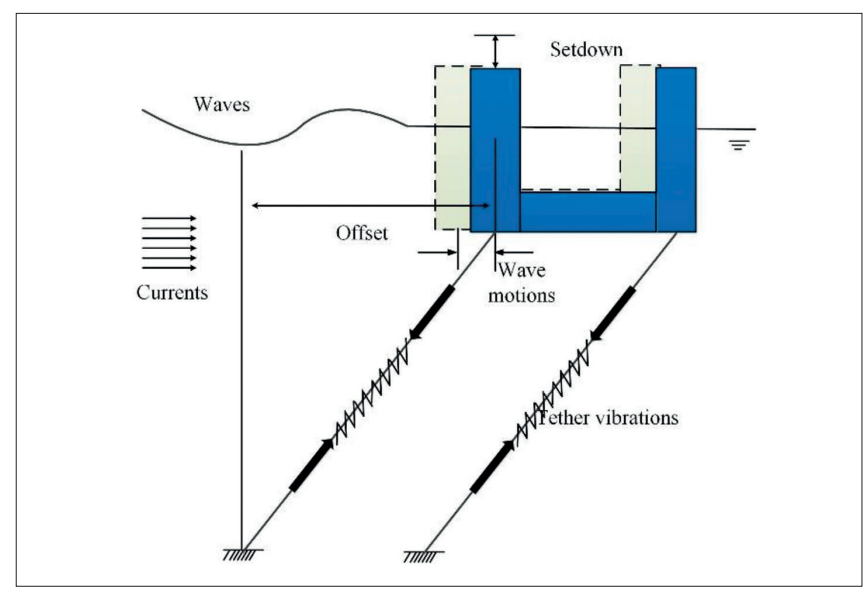

Fig. 1. Structure diagram of uncoupled TLP model

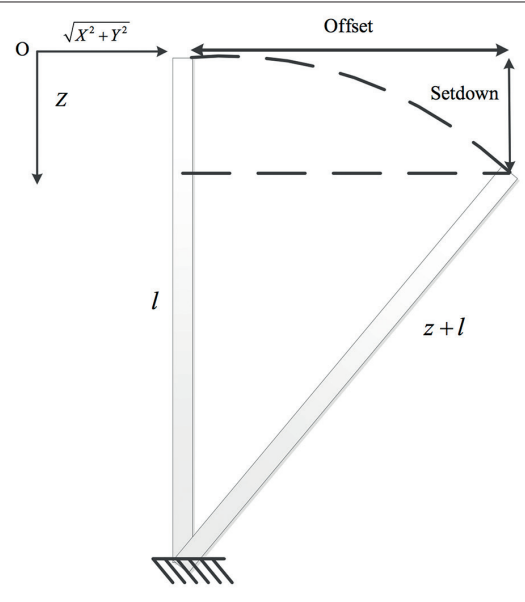

Fig. 2. Relationship between offset and setdown

When TLP is subjected to lateral force, the platform will have a horizontal offset. At the same time, the tether is in an inclined state. Because the tension leg has a narrow range of elasticity, the elongation of the tether is ignored when the stiffness matrix under the influence of the tendon is derived. The relationship satisfied by the platform's horizontal deviation and the vertical setdown phenomenon is shown in Fig. 2. Suppose that the horizontal offset of the platform has a component $\mathrm{x}$ on the $\mathrm{X}$-axis and a component $\mathrm{y}$ on the Y-axis, the vertical subsidence of the platform is $z=l\left[1-\sqrt{1-\left(\sqrt{X^{2}+Y^{2}} / l\right)^{2}}\right]$. Thus, the stiffness matrix coefficients of the platform under the influence of horizontal offset and vertical setdown are given, respectively.

As shown in Fig. 3, assuming that the displacement of the platform's longitudinal motion is $x_{1}$, the variation $\Delta T_{1}$ of TLP is:

$$
\Delta T_{1}=\left(\sqrt{\left(x_{1}+X\right)^{2}+l^{2}}-l\right) A E_{0} / l
$$

where $\Delta T_{1}$ is the axial force increment of the tether caused by the horizontal offset and surge motion of the platform; $l$ is the length of the tether; $A$ is the cross-sectional area of the tether; $E_{0}$ is the elastic modulus of the tether.
Affected by the horizontal offset of the platform, the phenomenon of vertical setdown occurs in TLP and is expressed by $z$.

$$
F_{V}+\rho g A_{w} z=F_{G}+\left(T_{0}+\Delta t\right)(l-z) / l
$$

Here, $F_{V}$ is the force due to buoyancy; $F_{G}$ is the force due to gravity. $A_{w}$ is the waterline surface area of the platform columns. $T_{0}$ is the pretension of the single tether. $\Delta t$ is the axial force increment of the tether caused by the vertical sinking of the platform.

By simplifying Eq. (3), the variation of tether caused by the vertical sinking of the platform can be obtained as follows:

$$
\Delta t=\left(T_{0}+\rho g A_{w} l\right) z /(l-z)
$$

In general, because $\Delta t$ is relatively small, it can be ignored when the analysis of a TLP mooring system is simulated.

The equilibrium forces of the platform in the $\mathrm{X}$ direction are:

$$
\bar{C}_{11} x_{1}=n_{t}\left(T_{0}+\Delta T_{1}+\Delta t\right) \sin \gamma_{x}
$$

where $n_{t}$ is the number of tethers (as shown in Fig. 3), and $\gamma_{x}$ is the angle between the position of the tensile tether and the initial position when the platform has surge motion. Additionally, the sine of $\gamma_{x}$ is defined as:

$$
\sin \gamma_{x}=\frac{x_{1}+X}{\sqrt{\left(x_{1}+X\right)^{2}+l^{2}}}
$$

Substituting equation $\gamma_{x}$ into Eq. (5), the restoring force coefficient of TLP surge motion can be obtained as follows:

$$
\bar{C}_{11}=\frac{n_{t}\left(T_{0}+\Delta T_{1}+\Delta t\right)\left(x_{1}+X\right)}{x_{1} \sqrt{\left(x_{1}+X\right)^{2}+l^{2}}}
$$

The equilibrium forces of the platform in the $\mathrm{Z}$ direction are:

$$
\bar{C}_{31} x_{1}=n_{t}\left(T_{0}+\Delta T_{1}+\Delta t\right) \cos \gamma_{x}+F_{G}
$$

Similarly, the equilibrium equation of the moments along the Y-axis can be obtained as follows:

$$
\bar{C}_{51}=-\frac{n_{t}\left(T_{0}+\Delta T_{1}+\Delta t\right)}{x_{1}} \sin \gamma_{x} \cdot \bar{h}
$$

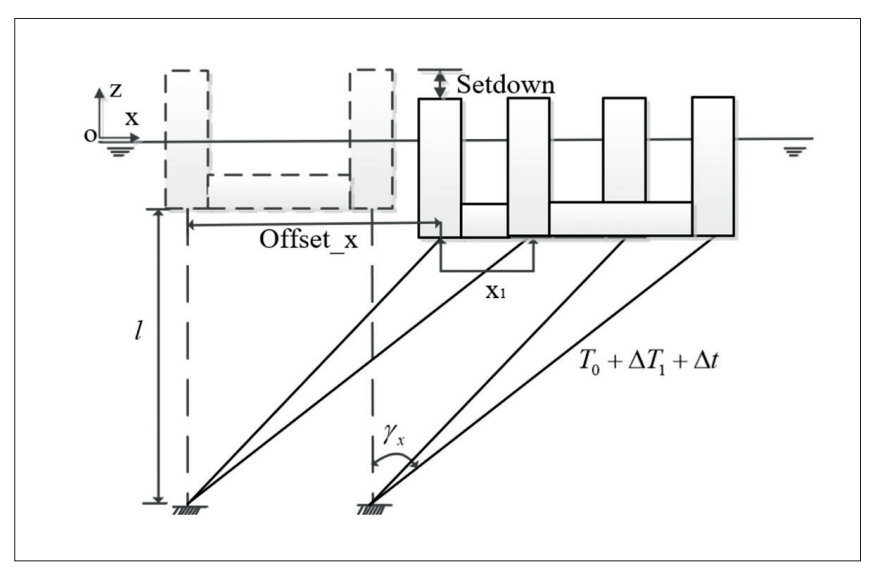

Fig. 3. Displacement in surge degree of freedom 


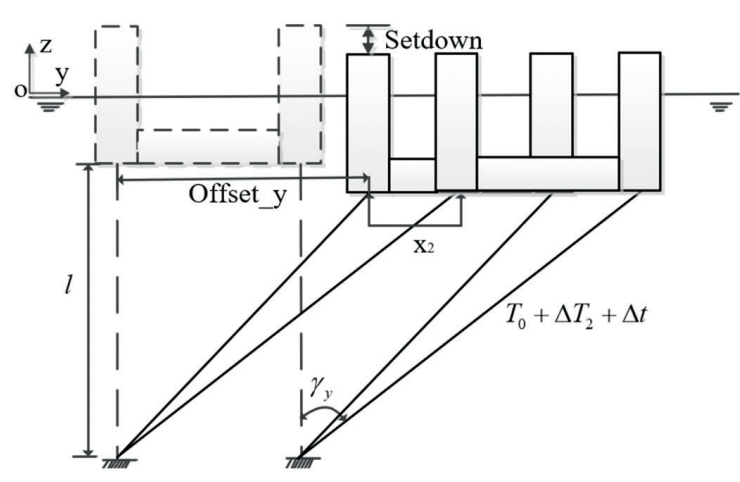

Fig. 4. Displacement in sway degree of freedom

where $\bar{h}$ is the height of gravity.

As shown in Fig. 4, assuming that the displacement of the platform's sway motion is $x_{2}$, then the change in tension of the tether caused by the combined action of the horizontal offset and the platform's swaying motion is:

$$
\Delta T_{2}=\left(\sqrt{\left(x_{2}+Y\right)^{2}+l^{2}}-l\right) A E_{0} / l
$$

Similarly, the equilibrium equation of forces on the Y-axis is as follows:

$$
\bar{C}_{22} x_{2}=n_{t}\left(T_{0}+\Delta T_{2}+\Delta t\right) \sin \gamma_{y}
$$

where $\Delta T_{2}$ is the axial force increment of the tether caused by the horizontal offset and sway motion of the platform. Meanwhile, $\gamma_{y}$ (shown in Fig. 4), is the angle between the position of the tensile tether and the initial position when the platform incurs sway motion. Taking the sine of $\gamma_{y}$ yields:

$$
\sin \gamma_{y}=\frac{x_{2}+Y}{\sqrt{\left(x_{2}+Y\right)^{2}+l^{2}}}
$$

The restoring force coefficient of sway motion is:

$$
\bar{C}_{22}=\frac{n_{t}\left(T_{0}+\Delta T_{2}+\Delta t\right)\left(x_{2}+Y\right)}{x_{2} \sqrt{\left(x_{2}+Y\right)^{2}+l^{2}}}
$$

In the same way, we can obtain the expressions shown as below:

$$
\begin{gathered}
\bar{C}_{32} x_{2}=n_{t}\left(T_{0}+\Delta T_{2}+\Delta t\right) \cos \gamma_{y}+F_{G} \\
\bar{C}_{42}=\bar{h} \bar{C}_{22}
\end{gathered}
$$

Assuming that the displacement of heave motion is $x_{3}$, then the restoring force coefficient is:

$$
\bar{C}_{33}=\left(n_{t} \frac{A E_{0}}{l}+A_{w}\right) \frac{\left(x_{3}+z\right)}{x_{3}}
$$

When the platform is in roll/pitch motion, the angle is assumed to be $x_{4}$. The compositions of roll/pitch motion consist of four parts: (1) stiffness due to static water surface; (2) stiffness due to changes in buoyancy and weight; (3) equivalent stiffness of the tethers; (4) stiffness due to vertical setdown of the platform. The restoring force coefficient of the roll/pitch motion is:

$$
\begin{aligned}
\bar{C}_{44}= & \frac{\rho g I_{x}}{\cos ^{3} x_{4}}+F_{V}\left(z_{B}-z_{E}\right)-F_{G}\left(z_{G}-z_{E}\right)+ \\
& \frac{E I_{x x}}{l} \cos ^{2} x_{4}+\rho g A_{w}\left(z_{B}-z_{E}\right) z \\
\bar{C}_{55}= & \frac{\rho g I_{y}}{\cos ^{3} x_{5}}+F_{V}\left(z_{B}-z_{E}\right)-F_{G}\left(z_{G}-z_{E}\right)+ \\
& \frac{E I_{y y}}{l} \cos ^{2} x_{5}+\rho g A_{w}\left(z_{B}-z_{E}\right) z
\end{aligned}
$$

where $I_{x}$ is the moment of inertia of the platform's waterplane in the X-axis; $I_{x x}$ is the cross-section moment of inertia of the platform in the X-axis; $I_{y}$ is the moment of inertia of the platform's waterplane in the Y-axis; $I_{y y}$ is the cross-section moment of inertia of the platform in the Y-axis; $z_{\beta}$ is the vertical coordinate of the buoyancy centre of the platform; $z_{E}$ is the vertical coordinate of the top of the tether; $z_{G}$ is the vertical coordinate of the centre of gravity of the platform.

When the platform incurs yaw motion, its angle is assumed to be $x_{6}$. The displacement of the tether in the horizontal plane is given in Fig. 5, which is mainly composed of two parts. The first part is the displacement caused by the horizontal offset of the platform, and the second part is the displacement caused by the yaw movement of the platform. Then, the elongation of the tether is:

$$
l_{1}=\sqrt{\left(\sqrt{X^{2}+Y^{2}}+x_{6} \sqrt{a^{2}+b^{2}}\right)^{2}+l^{2}}
$$

where $a$ and $b$ are half of the distance between the centre points of the columns, shown in Fig. 5.

Based on Eq. (19), when the platform incurs simultaneous horizontal offset and yaw motion, the variation of the tether is as follows:

$$
\Delta T_{6}=\frac{A E_{0}}{l}\left(l_{1}-l\right)
$$

The balance forces of the platform in the vertical plane are:

$$
\bar{C}_{36} x_{6}=n_{t}\left(T_{0}+\Delta T_{6}+\Delta t\right)+F_{G}-F_{V}
$$

By establishing the balance equation of the moments in the $\mathrm{Z}$-axis of the platform, the restoring force coefficient of sway motion can be obtained:

$$
\bar{C}_{66}=n_{t}\left(T_{0}+\Delta T_{6}+\Delta t\right) \cdot \frac{a^{2}+b^{2}}{\sqrt{l^{2}+x_{6}^{2}\left(a^{2}+b^{2}\right)}}
$$

By calculating the TLP restoring force coefficient matrix and applying the Runge-Kutta method to the differential motion equation of the platform, the motion equation of TLP under wave action can be obtained. Fig. 6 shows the flow chart of the uncoupled TLP model. 


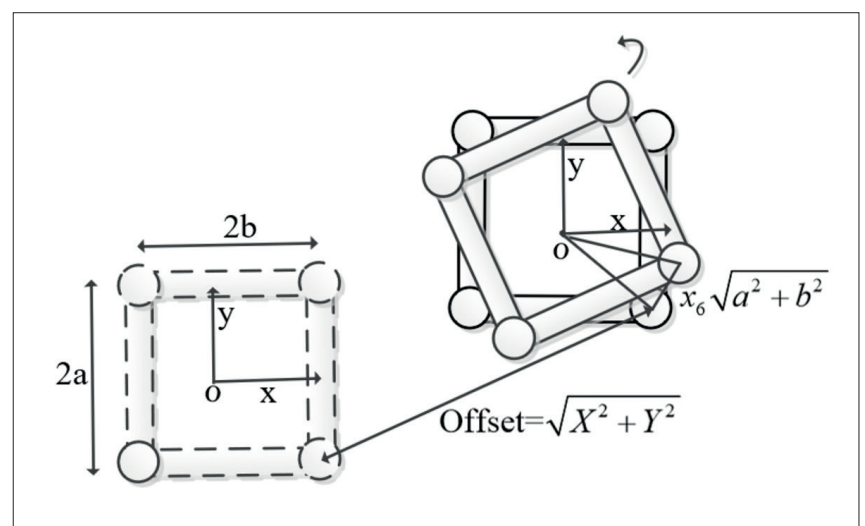

Fig. 5. Displacement in yaw degree of freedom

\section{MATHEMATICAL COUPLED TLP MODEL}

Fig. 7 illustrates the structure of the uncoupled TLP model. In order to study the TLP time-domain motion responses, the coupled condition of the tether vibration and platform motion is considered, and the coupled TLP model is used to conduct numerical prediction research. In the research process, the following two factors are mainly considered in the analysis of the TLP motion responses:

1) The coupled effect between axial and transverse vibration of the tether;

2) The influence of the coupled effect of the platform motion.

\section{TIME DOMAIN MOTION EQUATION OF TLP}

When the time domain numerical simulation of TLP is carried out in this paper, the platform is regarded as a rigid

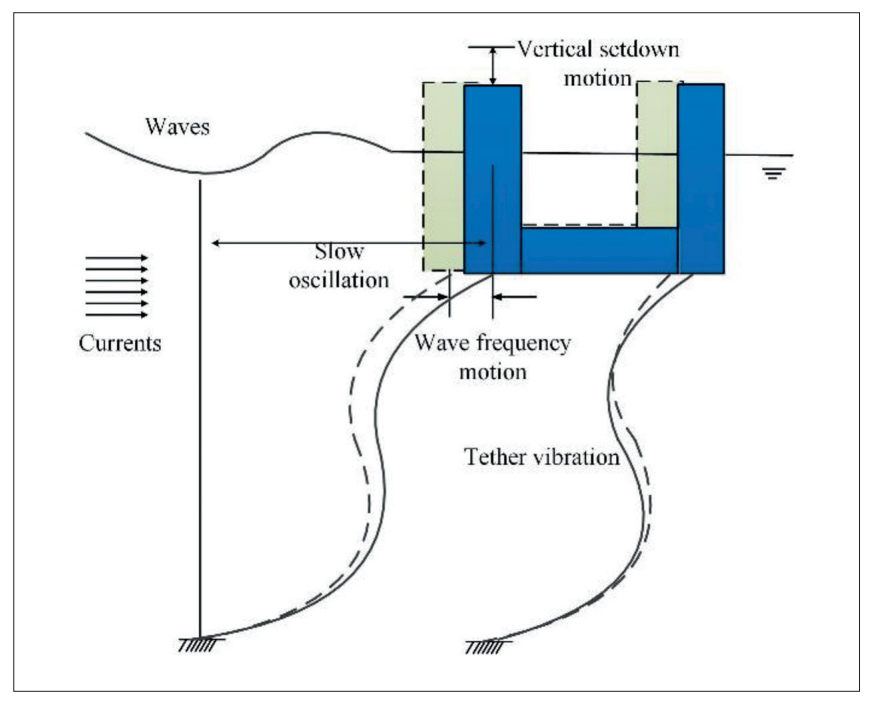

Fig. 7. Structure diagram of coupled TLP model

body, and the coupled motion Eqs. (20) and (21) of TLP considering the tether vibration influence is given.

$$
\begin{gathered}
\left(M_{i j}+\mu_{i j}\right) \ddot{x}_{j}(t)+K_{i j} x_{j}(t)+C_{i j} x_{j}(t)= \\
F_{i \text { Wave }}(t)+F_{i}+F_{V}-F_{G}
\end{gathered}
$$

In Eq. (23), $\bar{C}_{i j}$ is the coefficient of restoring force matrix; $F_{i \text { Wave }}$ is the force exerted by the waves on the platform; $F_{i}$ is the force exerted by the tethers on the platform; $F_{V}$ is the buoyancy of the platform; $F_{G}$ is the gravity of the platform.

The tether is attached to the platform at $z=L$ and the seabed at $z=0$; the function $w_{3}(z, t)$ is used to express the axial vibration of the tether. The axial vibration of the tether is:

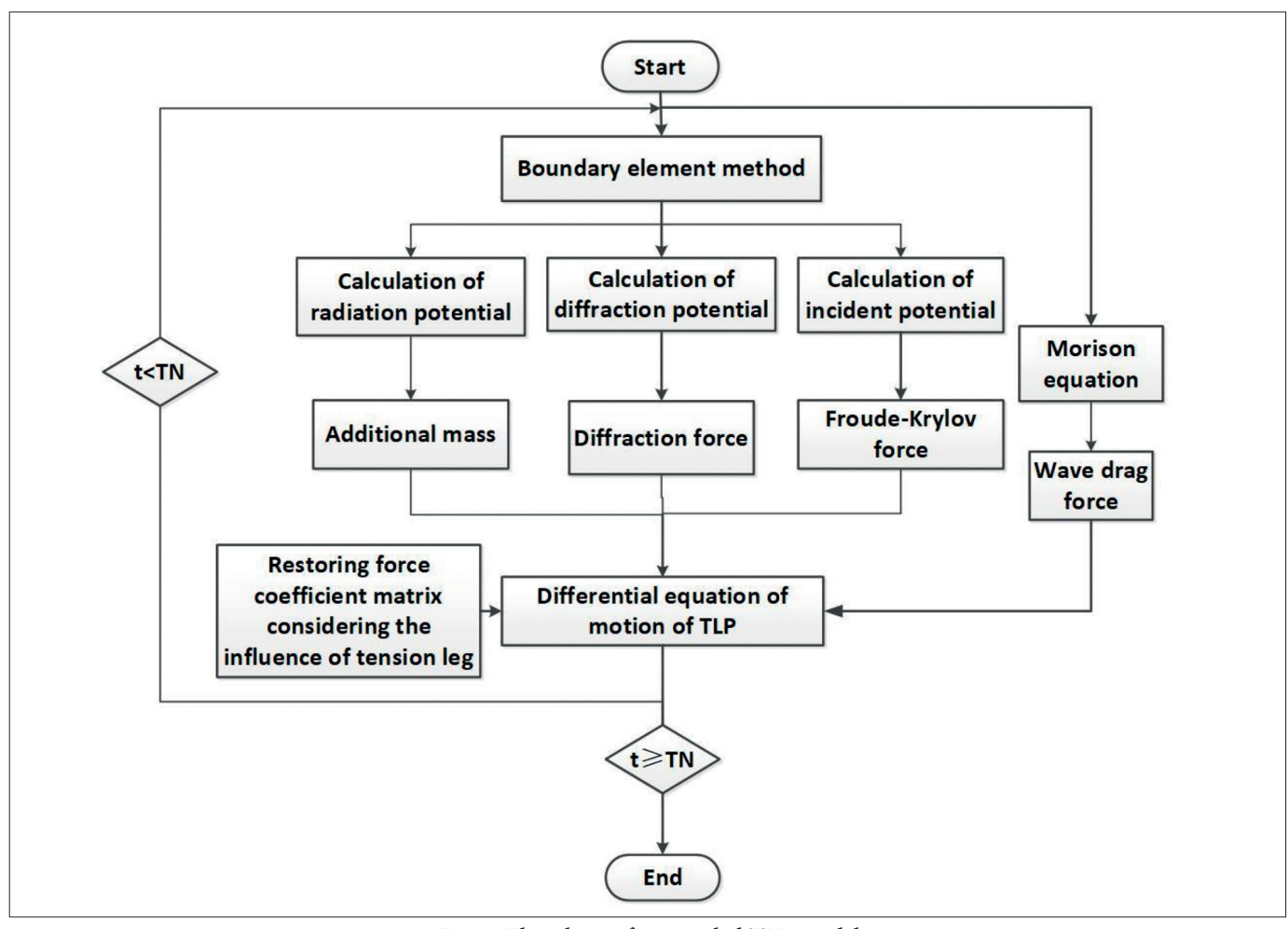

Fig. 6. Flowchart of uncoupled TLP model 


$$
\rho A_{0} \ddot{w}_{3}(z, t)-\left(E A_{0}\left(w_{3}^{\prime}(z, t)+\frac{1}{2} w_{r}^{\prime}(z, t)^{2}\right)\right)=f_{3}(z, t)
$$

where $\rho$ is the material density of the tether; $w_{r}(z, t)$ is the radial motion; $f_{3}(z, t)$ is the distributed force-per-unit-length along the axial direction of the tether, and its expression is:

$$
f_{0}(z, t)= \begin{cases}-\rho g A_{0}+\rho_{f} g A_{f} & 0<z<d \\ \rho g A_{0} & d<z<l\end{cases}
$$

There is no displacement at $z=0$, and its boundary condition is:

$$
w_{3}(0, t)=0, w_{3}^{\prime}(0, t)=0
$$

At $z=L$, the force of the tether on the platform is

$$
F_{L 3}(l, t)=-n_{t}\left(T_{0}+E A_{0} w_{3}(l, t)\right)
$$

Taking the platform itself as the research object, the external forces acting on the platform mainly include gravity, buoyancy, wave forces, and tether forces, and combined with Eq. (23), the differential equation of heave motion is obtained:

$$
\begin{gathered}
\left(M_{33}+\mu_{33}\right) \ddot{w}_{3}(l, t)+C_{3 i} w_{i}(t)= \\
F_{3 \text { Wave }}(t)+F_{L 3}(l, t)+F_{V}-F_{G}
\end{gathered}
$$

In conclusion, the numerical solution of heave motion is achieved by combining Eqs. (24), (27), and (28). Considering the fact that the coupled TLP model takes the influence of axial and transverse vibrations of the tethers into account, it is necessary to solve the governing equations of the platform's surge and sway motions before solving the governing equation of TLP heave motion.

The equation of radial vibration of the tether in the $\mathrm{x}$-direction is:

$$
\begin{gathered}
\rho A \ddot{w}_{1}(z, t)-\left(E A_{0}\left(w_{3}^{\prime}(z, t)+\frac{1}{2} w_{1}^{\prime}(z, t)^{2}\right)\right)+ \\
\left(E I_{0} w_{1}^{\prime \prime}(z, t)\right)^{\prime \prime}-\rho I_{0} \ddot{w}_{1}^{\prime \prime}(z, t)=f_{1}(z, t)
\end{gathered}
$$

The transverse force $f_{1}(z, t)$ is mainly produced by the action of waves and currents and formulated using the Morison equation. Suppose the angle between the current and the $\mathrm{x}$-direction is $\theta_{0}$ and we get:

$$
\begin{gathered}
f_{1}=-C_{A} \rho_{f} A_{f}\left(-\ddot{w}_{3} w_{1}^{\prime \prime}+\ddot{w}_{1}\right)+C_{M} \rho_{f} A_{f}\left(\dot{\varphi}_{1}-\varphi_{3} w_{1}^{\prime}\right)+ \\
C_{D} \rho_{f} r_{\text {outer }}\left(\dot{\varphi}_{1}-\varphi_{3} w_{1}^{\prime}+\dot{w}_{3} \varphi_{1}^{\prime}-\dot{w}_{1}+U_{c} \cos \theta_{0}\right) \\
\left|\dot{\varphi}_{1}-\varphi_{3} w_{1}^{\prime}+\dot{w}_{3} \varphi_{1}^{\prime}-\dot{w}_{1}+U_{c} \cos \theta_{0}\right|
\end{gathered}
$$

where $U_{c}$ is the current velocity, $C_{A}$ is the added mass coefficient, $C_{D}$ is the drag coefficient, and $C_{M}$ is the inertia coefficient. $\varphi_{1}$ and $\varphi_{3}$ are velocities of fluid particles caused by waves in the $\mathrm{x}$-axis and $\mathrm{z}$-axis, respectively, given by:

$$
\begin{aligned}
& \varphi_{1}=\frac{\pi H}{T} \frac{\cosh (k z)}{\sinh (k d)} \cos (k r-\omega t) \cos \theta_{0} \\
& \varphi_{3}=\frac{\pi H}{T} \frac{\sinh (k z)}{\sinh (k d)} \sin (k z-\omega t)
\end{aligned}
$$

where $H$ is the wave height; $T$ is the wave period; $k$ is the wave number; $\omega$ is the wave frequency; $d$ is the depth of water. The boundary condition of the tether is:

$$
w_{1}(0, t)=0, w_{1}^{\prime}(0, t)=0
$$

The force of tether on the platform at $x=L$ is:

$$
F_{L 1}(l, t)=n_{t} E A_{0}\left(w_{3}^{\prime}+\frac{1}{2} w_{1}^{\prime 2}\right) w_{1}^{\prime}-n_{t} E I_{0} w_{1}^{\prime \prime \prime}
$$

The differential equation of surge motion is:

$$
\left(M_{11}+\mu_{11}\right) \ddot{w}_{1}(l, t)+C_{1 i} w_{i}(l, t)=F_{1 \text { Wave }}(t)+F_{L 1}(l, t)
$$

In conclusion, the numerical simulation of TLP surge motion can be solved by combining Eqs. (29), (34), and (35).

The vibration equation of radial motion of the tether in the $y$-direction is:

$$
\begin{gathered}
\rho A \ddot{w}_{2}(z, t)-\left(E A_{0}\left(w_{3}^{\prime}(z, t)+\frac{1}{2} w_{2}^{\prime}(z, t)^{2}\right) w_{2}^{\prime}(z, t)\right)+ \\
\left(E I_{0} w_{2}^{\prime \prime}(z, t)\right)^{\prime \prime}-\rho I_{0} \ddot{w}_{2}^{\prime \prime}(z, t)=f_{2}(z, t)
\end{gathered}
$$

The transverse force in the $y$-direction is:

$$
\begin{gathered}
f_{2}=-C_{A} \rho_{f} A_{f}\left(-\ddot{w}_{3} w_{2}^{\prime}+\ddot{w}_{2}\right)+C_{M} \rho_{f} A_{f}\left(\dot{\varphi}_{2}-\varphi_{3} w_{2}^{\prime}\right)+ \\
C_{D} \rho_{f} r_{\text {outer }}\left(\dot{\varphi}_{2}-\varphi_{3} w_{2}^{\prime}+\dot{w}_{3} \varphi_{2}^{\prime}-\dot{w}_{2}+U_{c} \sin \theta_{0}\right) \\
\left|\dot{\varphi}_{2}-\varphi_{3} w_{2}^{\prime}+\dot{w}_{3} \varphi_{2}^{\prime}-\dot{w}_{2}+U_{c} \sin \theta_{0}\right|
\end{gathered}
$$

where $\varphi_{2}$ is the velocity of fluid particles caused by waves in the $\mathrm{y}$-axis, given by:

$$
\varphi_{2}=\frac{\pi H}{T} \frac{\cosh (k z)}{\sinh (k d)} \cos (k r-\omega t) \sin \theta_{0}
$$

The boundary condition of the tether is:

$$
w_{2}(0, t)=0, w_{2}^{\prime}(0, t)=0
$$

The force of tether on the platform at $x=L$ is:

$$
F_{L 2}(l, t)=n_{t} E A_{0}\left(w_{3}^{\prime}+\frac{1}{2} w_{2}^{\prime 2}\right) w_{2}^{\prime}-n_{t} E I_{0} w_{2}^{\prime \prime \prime}
$$

The differential equation of sway motion is:

$$
\left(M_{22}+\mu_{22}\right) \ddot{w}_{2}(l, t)+C_{2 i} w_{i}(l, t)=F_{2 \text { Wave }}(t)+F_{L 2}(l, t)
$$

In conclusion, the numerical simulation of the sway motion can be solved by combining Eqs. (36), (40), and (41).

Longitudinal tether excitations will impart disturbances 
in the pitch $(\theta)$ degree of freedom. Pitch motion is defined as an angular displacement occurring about the y-axis, shown in Fig. 8a. The equation describing the pitch displacement is written as:

$$
\begin{gathered}
\left(M_{55}+\mu_{55}\right) \ddot{w}_{5}(l, t)+C_{5 i} w_{i}(l, t)= \\
F_{5 \text { Wave }}(t)+F_{L 5}(l, t)+F_{V} e_{5}
\end{gathered}
$$

where $F_{5 \text { wave }}$ is the moment of a wave to the platform in the $\mathrm{y}$-axis; $F_{L 5}$ is the moment of tether to the platform in the y-axis; $F_{V}$ is the buoyancy of the platform; $e_{5}$ is the perpendicular distance of the new centre of buoyancy from the axis passing through theCG(centre of gravity); $e_{5}=\bar{h} \sin w_{5}$, where $\bar{h}$ is the height of the centre of gravity.

Longitudinal tether excitations will also impart disturbances in the roll $(\varphi)$ degree of freedom. Roll motion is defined as an angular displacement occurring about the x-axis, shown in Fig. 8a. The equation describing the roll displacement is written as:

$$
\begin{gathered}
\left(M_{44}+\mu_{44}\right) \ddot{w}_{4}(l, t)+C_{4 i} w_{i}(l, t)= \\
F_{4 \text { Wave }}(t)+F_{L 4}(l, t)+F_{V} e_{4}
\end{gathered}
$$

where $F_{4 \text { wave }}$ is the moment of a wave to the platform in the x-axis; $F_{L 4}$ is the moment of the tether to the platform in the $\mathrm{x}$-axis; $e_{4}$ is the perpendicular distance of the new centre of buoyancy from the $\mathrm{x}$-axis passing through the CG, with equation $e_{4}=\bar{h} \sin w_{4}$.

The yaw motion of TLP is mainly caused by the wave moment and the moment acted upon the tethers in the $\mathrm{z}$-axis direction, shown in Fig. 8b. The differential equation of motion satisfied by the angular displacement of the platform under the action of the moments in the $\mathrm{z}$-axis direction is:

$$
\left(M_{66}+\mu_{66}\right) \ddot{w}_{6}(l, t)+C_{6 i} w_{i}(l, t)=F_{6 \text { Wave }}(t)+F_{L 6}(l, t)
$$

where $F_{6 \text { Wave }}$ is the moment of a wave to the platform in the z-axis; $F_{L 6}$ is the moment of the tether to the platform in the $\mathrm{z}$-axis.

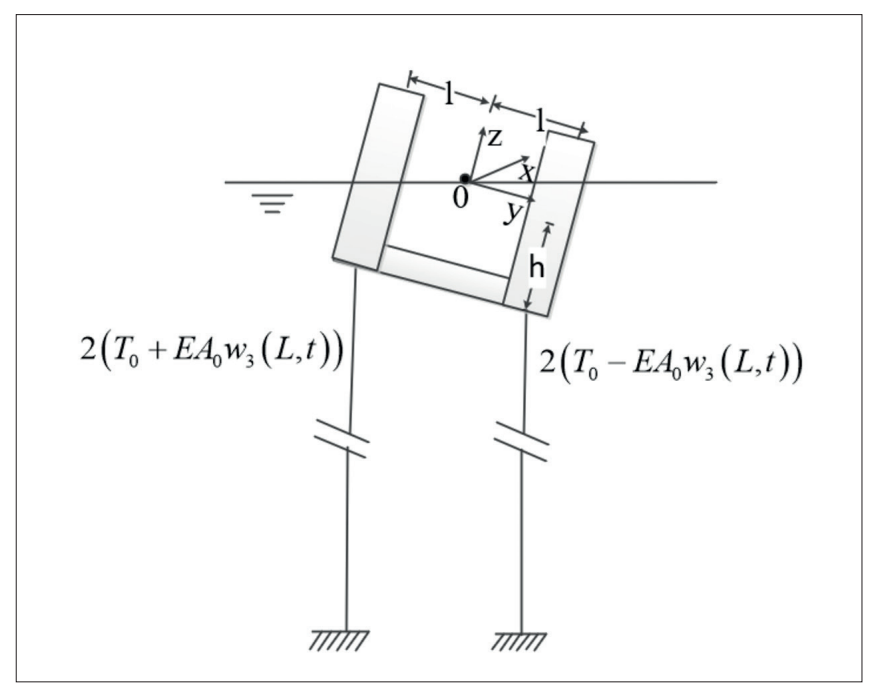

Fig. 8a. The motions diagram of TLP: pitch/roll motion

\section{CALCULATION METHOD OF THE COUPLED TLP MODEL}

Taking surge motion as an example, the boundary conditions of the tether are:

$$
\begin{aligned}
& \text { At } \mathrm{z}=0 \text {, } \\
& w_{1}(0, t)=0, w_{1}^{\prime}(0, t)=0 \\
& \text { At } \mathrm{z}=\mathrm{L} \text {, } \\
& F_{L 1}(l, t)=n_{t} E A_{0} N(l) w_{1}^{\prime}(l, t)-n_{t} E I_{0} w_{1}^{\prime \prime \prime}(l, t) \\
& F_{L 2}(l, t)=n_{t} E A_{0} N(l) w_{2}^{\prime}(l, t)-n_{t} E I_{0} w^{\prime \prime \prime}{ }_{1}(l, t) \\
& F_{L 3}(l, t)=-n_{t}\left(T_{0}+E A_{0} w_{3}(l, t)\right) \\
& F_{L 4}(l, t)=-n_{t}\left(h T_{0} w_{4}(l, t)+a E A_{0} w_{1}(l, t)\right) \\
& F_{L 5}(l, t)=-n_{t}\left(h T_{0} w_{5}(l, t)+a E A_{0} w_{2}(l, t)\right) \\
& F_{L 6}(l, t)=-\sqrt{2} n_{t} a T_{0} w_{1}(l, t)
\end{aligned}
$$

where $F_{L i}(i=1, \ldots, 6)$ are the forces of the tethers on the platform with six degrees of freedom. $N(z)$ is the axial force of the beam, given by $N(z)=E A_{0}\left(w_{3}^{\prime}(z, t)+\frac{1}{2} w_{1}^{\prime}(z, t)^{2}\right)$.

If the system is vibrating in its ith mode, then the response of the system would be as

$$
w(z, t)=\varphi_{i}(z) \exp \left(I \omega_{i} t\right)
$$

where $I$ is the imaginary unit, $\omega_{i}$ is the ith normalised natural frequency, and $\varphi_{i}(x)$ is the ith normalised transverse mode shape of the beam.

By dropping the axial, damping, non-linear, and nonhomogenous terms in the governing PDE and boundary conditions of the transverse motion, and using Eq. (29), one would get:

$$
E I_{0} \varphi_{i}^{(4)}(z)-\left(N(z) \varphi_{i}^{\prime}(z)\right)^{\prime}-\rho A_{0} \omega_{i}^{2} \varphi_{i}(z)=0
$$

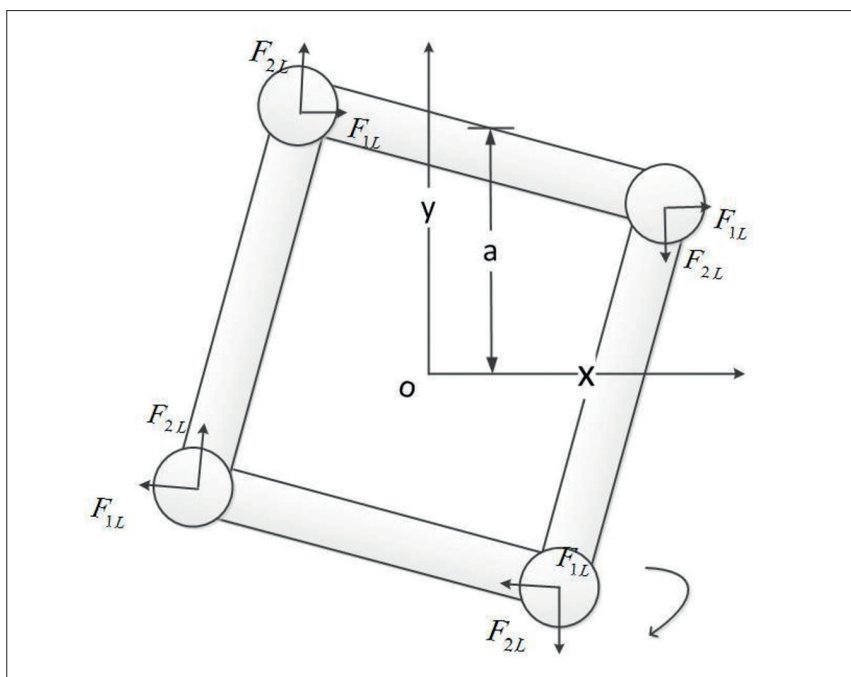

Fig. 8b. The motions diagram of TLP: yaw motion 
and $\varphi_{i}$ satisfies the boundary conditions of $\varphi_{i}(0)=0, \varphi_{i}^{\prime}(0)=0$.

Eq. (48) is rewritten into the form of first-order equations, namely:

$$
\frac{d \vec{Y}_{i}(z)}{d z}=[A]_{\left(z, \omega_{i}\right)} \vec{Y}_{i}(z)
$$

where $\vec{Y}_{i}(z)=\left[\varphi_{i}(z) \varphi_{i}^{\prime}(z) \varphi_{i}^{\prime \prime}(z) \varphi^{\prime \prime \prime}{ }_{i}(z)\right]^{T}$.

$$
\text { The matrix }[A]_{\left(z, \omega_{i}\right)}=\left[\begin{array}{cccc}
0 & 1 & 0 & 0 \\
0 & 0 & 1 & 0 \\
0 & 0 & 0 & 1 \\
\frac{\rho A_{0} \omega_{i}^{2}}{d z} & \frac{N^{\prime}(z)}{E I_{0}} & \frac{N(z)}{E I_{0}} & 0
\end{array}\right]
$$

According to the definition of the first derivative, the approximate expression of Eq. is:

$$
\frac{d \vec{Y}_{i}(z)}{d z}=\frac{\vec{Y}_{i}(z+\Delta z)-\vec{Y}_{i}(z)}{\Delta z}
$$

where $\Delta z$ is the increment of the tether at the position $z$. Substitute Eq. (50) into Eq. (49) and one gets:

$$
\vec{Y}_{i}(z+\Delta z)=\left[[A]_{\left(z, \omega_{i}\right)} \Delta z+I_{4 \times 4}\right]_{i} \vec{Y}(z)
$$

The tether is dispersed into $n$ equal parts, as shown in Fig. 9. The nodes are numbered from 0 to $N$.

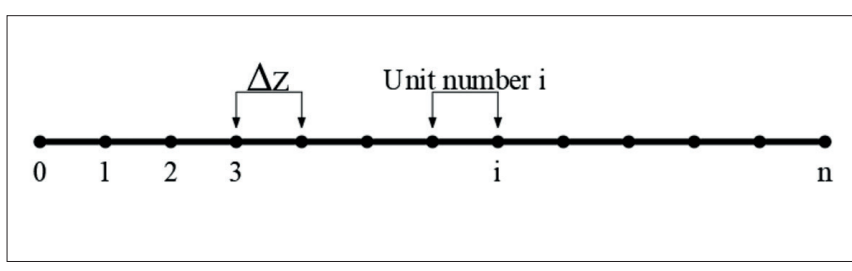

Fig. 9. Nodes of tether numbering scheme

By applying the above tether discrete method, the motion equation at node $k$ can be expressed as:

$$
\vec{Y}_{i}(k \Delta z)=\prod_{j=1}^{k}\left[[A]_{\left(k-j, \omega_{i}\right)} \Delta z+I_{4 \times 4}\right]_{i} \vec{Y}(0)
$$

When $k=n$, the expression of the end node of the tether is:

$$
\vec{Y}_{i}(l)=\prod_{j=1}^{n}\left[[A]_{\left(n-j, \omega_{i}\right)} \Delta z+I_{4 \times 4}\right]_{i} \vec{Y}(0)
$$

Namely,

$$
\left[\begin{array}{c}
\varphi_{i}(l) \\
\varphi_{i}^{\prime}(l) \\
\varphi_{i}^{\prime \prime}(l) \\
\varphi_{i}^{\prime \prime \prime}(l)
\end{array}\right]=\left[\begin{array}{lllll}
b_{1,1}\left(\omega_{i}\right) & b_{1,2}\left(\omega_{i}\right) & b_{1,3}\left(\omega_{i}\right) & b_{1,4}\left(\omega_{i}\right) \\
b_{i, 1}\left(\omega_{i}\right) & b_{2,2}\left(\omega_{i}\right) & b_{2,3}\left(\omega_{i}\right) & b_{2,4}\left(\omega_{i}\right) \\
b_{, 3,1}\left(\omega_{i}\right) & b_{3,2}\left(\omega_{i}\right) & b_{3,3}^{2}\left(\omega_{i}\right) & b_{3,4}^{2}\left(\omega_{i}\right) \\
b_{4,1}^{3,}\left(\omega_{i}\right) & b_{4,2}\left(\omega_{i}\right) & b_{4,3}^{3,3}\left(\omega_{i}\right) & b_{4,4}^{3,}\left(\omega_{i}\right)
\end{array}\right]\left[\begin{array}{c}
0 \\
0 \\
\varphi_{i,}^{\prime \prime}(0) \\
\varphi_{i}^{\prime \prime \prime}(0)
\end{array}\right]
$$

where $\varphi_{i}^{\prime \prime}(l)$ can be obtained by the force of the tether on the platform, and:

$$
F_{L 4}(l, t)=n_{t} E I_{0} w_{1}^{\prime \prime}(l, t)=n_{t} E I_{0} \varphi_{i}^{\prime \prime}(l) \exp \left(I \omega_{i} t\right)
$$

Similarly, $\varphi^{\prime \prime \prime}{ }_{i}(l)$ can be obtained by the force $F_{L 1}(l, t)$ exerted by the tether on the platform. Thus, $\varphi_{i}^{\prime \prime}(l)$ and $\varphi_{i}^{\prime \prime \prime}(l)$ can be expressed by the interaction forces $F_{L 1}(l, t)$ and $F_{L 4}(l, t)$, respectively. Then, the expression of Eq. (51) is:

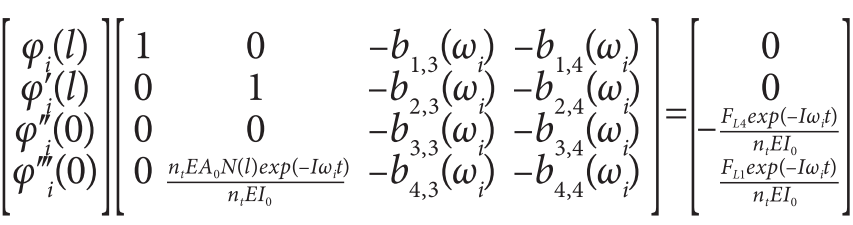

According to the axial motion Eq. (24) of the tether, we can obtain:

$$
\rho A_{0} \ddot{w}_{3}(l, t)-N^{\prime}(l)=f_{3}(l, t)
$$

The governing Eqs. (55) and (56) of the tethers are solved together with the surge motion Eq. (28) and the heave motion Eq. (35), and the time history curves of the surge motion and heave motion of TLP can be obtained. Similarly, the solution of sway motion is similar to the surge motion. After the time history curves of TLP's surge, sway, and heave motions are obtained, the interaction forces between the tethers and the platform, as shown in Eq. (46), are inserted into Eqs. (42), (43), and (44), and solved in parallel. The time history curves of pitch, roll, and yaw motions are obtained.

The calculation flow chart of the coupled TLP model is shown in Fig. 10.

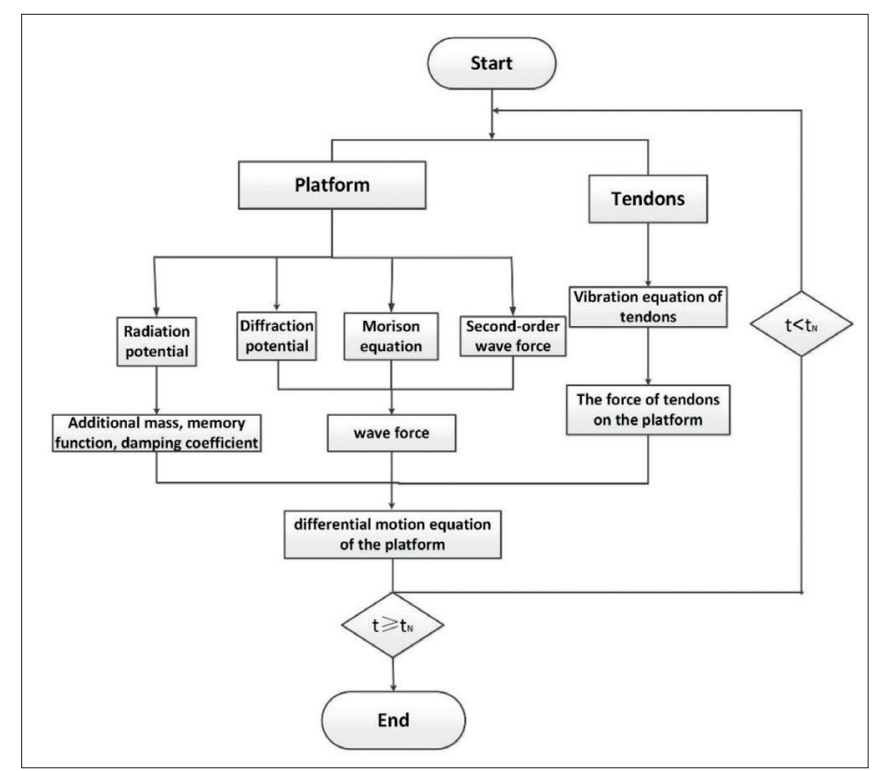

Fig. 10. Time domain coupled motion flow chart of TLP

\section{COMPARATIVE ANALYSIS OF THE COUPLED TLP MODEL AND THE UNCOUPLED TLP MODEL}

The four corners of the platform are connected by four groups of tethers, and each group is composed of two tethers, as shown in Fig. 11. The calculation parameters of the platform are shown in Table 1; the number of surface elements of TLP is 3105 , and the time step is $0.05 \mathrm{~s}$. Table 2 shows the parameters of the tether. The flow parameters of the regular waves are shown in Table 3. We will use the uncoupled TLP numerical 
program and the coupled TLP numerical program, respectively, to calculate the mathematical model in this paper.

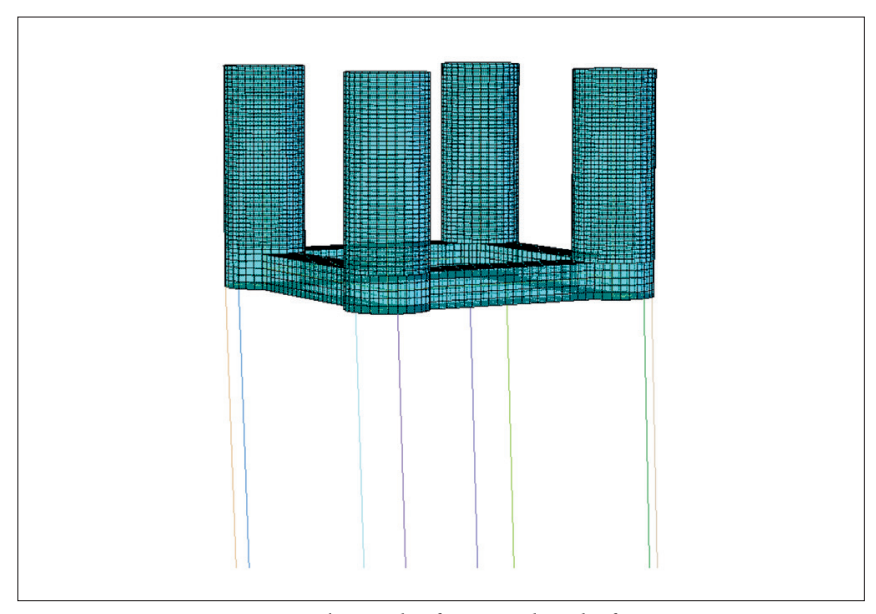

Fig. 11. The mesh of tension leg platform

Tab. 1. ISSC TLP calculation parameters

\begin{tabular}{|c|c|}
\hline Parameters & Values \\
\hline Depth of column $(\mathrm{m})$ & 31.3 \\
\hline Length of pontoon $(\mathrm{m})$ & 60.0 \\
\hline Diameter of side column $(\mathrm{m})$ & 16.0 \\
\hline Total platform mass (including vertical tension) $(\mathrm{kg})$ & $3.466 \mathrm{e} 7$ \\
\hline Number of tethers & 8 \\
\hline The moment of inertia in the X direction $\left(\mathrm{kg} \cdot \mathrm{m}^{2}\right)$ & $8.45 \mathrm{e} 10$ \\
\hline The moment of inertia in the $\mathrm{Y}$ direction $\left(\mathrm{kg} \cdot \mathrm{m}^{2}\right)$ & $8.45 \mathrm{e} 10$ \\
\hline The moment of inertia in the $\mathrm{Z}$ direction $\left(\mathrm{kg} \cdot \mathrm{m}^{2}\right)$ & $1.02 \mathrm{e} 11$ \\
\hline
\end{tabular}

Tab. 2. Tether parameters

\begin{tabular}{|c|c|}
\hline Parameters & Values \\
\hline Tether length $(\mathrm{m})$ & 600 \\
\hline Inner diameter of tether $(\mathrm{m})$ & 0.2501 \\
\hline Outer diameter of tether $(\mathrm{m})$ & 0.9 \\
\hline Total pretension $(\mathrm{N})$ & $1 \mathrm{e} 8$ \\
\hline Material density $\left(\mathrm{kg} \cdot \mathrm{m}^{-3}\right)$ & 6000 \\
\hline Elasticity modulus $(\mathrm{GPa})$ & 200 \\
\hline
\end{tabular}

Tab. 3. Flowfield parameters

\begin{tabular}{|c|c|}
\hline Parameters & Values \\
\hline Wave length $(\mathrm{m})$ & 156 \\
\hline Wave height $(\mathrm{m})$ & 10 \\
\hline Wave period (s) & 10 \\
\hline Coefficient of inertia force & 2 \\
\hline Coefficient of mass & 1 \\
\hline Coefficient of drag force & 1 \\
\hline Depth (m) & 430 \\
\hline Angle (degrees) & $60^{\circ}$ \\
\hline
\end{tabular}

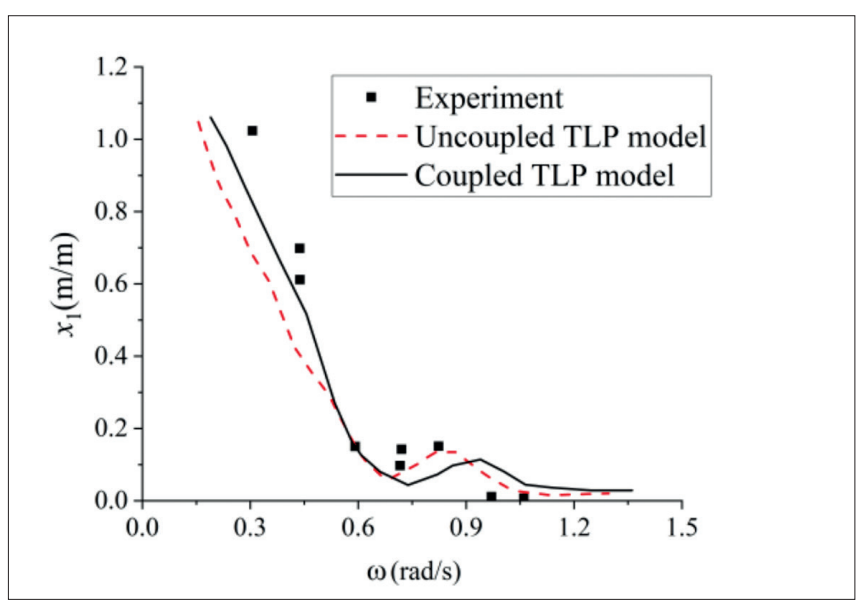

Fig. 12a. RAO for TLP: surge motion

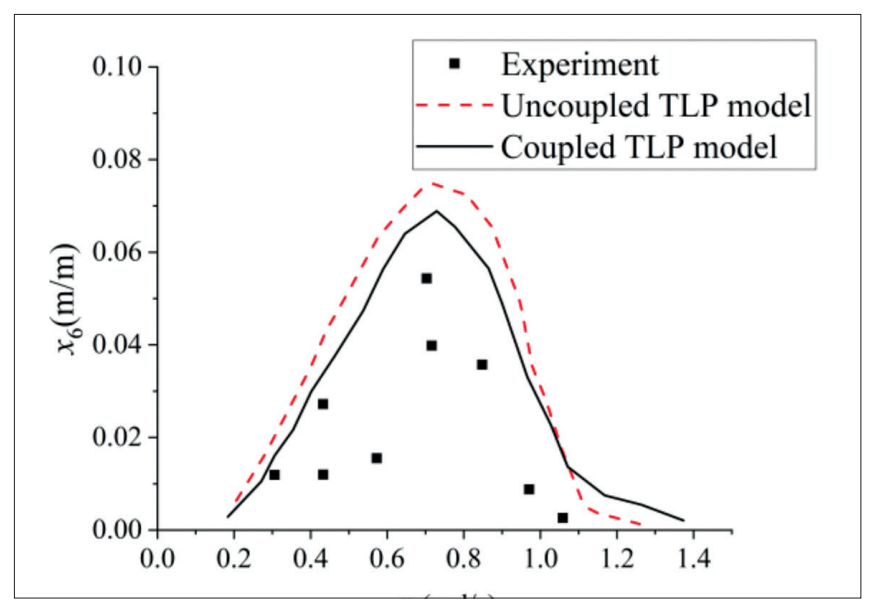

Fig. 12b. RAO for TLP: yaw motion

In order to verify the accuracy of the calculation results, the uncoupled TLP model and the coupled TLP model are respectively used to calculate the RAO motion response of the ISSC TLP platform, and the calculated results are compared with the experimental results of Tan [20]. Tan's model test was carried out in a seakeeping basin with a water depth of $450 \mathrm{~m}$. The experimental simulation of the RAO test was carried out for the model respectively. By comparison, it can be concluded that the coupled TLP model is closer to the experimental results than the uncoupled TLP model. The main reasons for the error between the theoretical analysis results and the experimental results include: the average wet surface theory is applied to the wet surface of the floating body when the plane element method is used for model analysis, but the actual wet surface changes with time.

Fig. 13 shows the comparative results of the motion responses in the regular waves using different methods. Among them, the dotted line represents the uncoupled TLP model, which assumes the tether as a massless spring structure. The solid line stands for the coupled TLP model calculation method, in which the current velocity is $0 \mathrm{~m} / \mathrm{s}$, and the wave direction angle is $60^{\circ}$. By studying the statistical results in Table 4, it can be concluded that the vibration frequency of the uncoupled TLP model is similar to that of the coupled TLP model, and the vibration amplitude of the coupled TLP model 


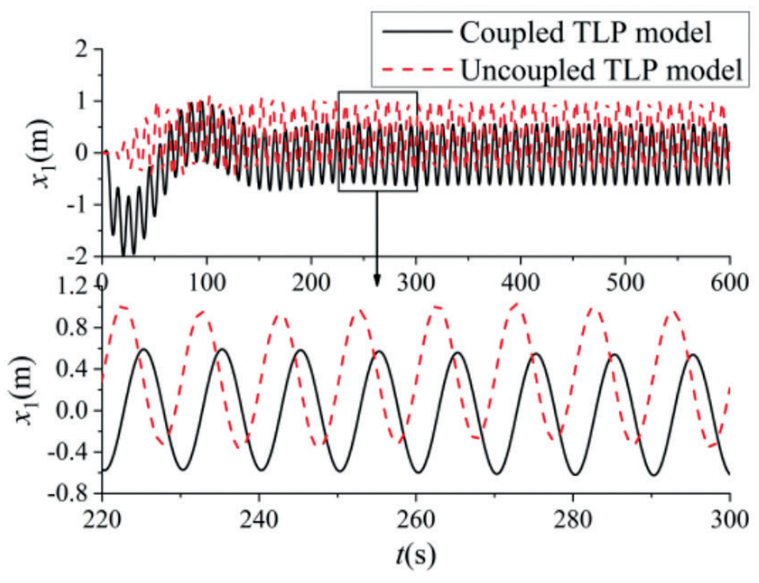

a) Surge motion

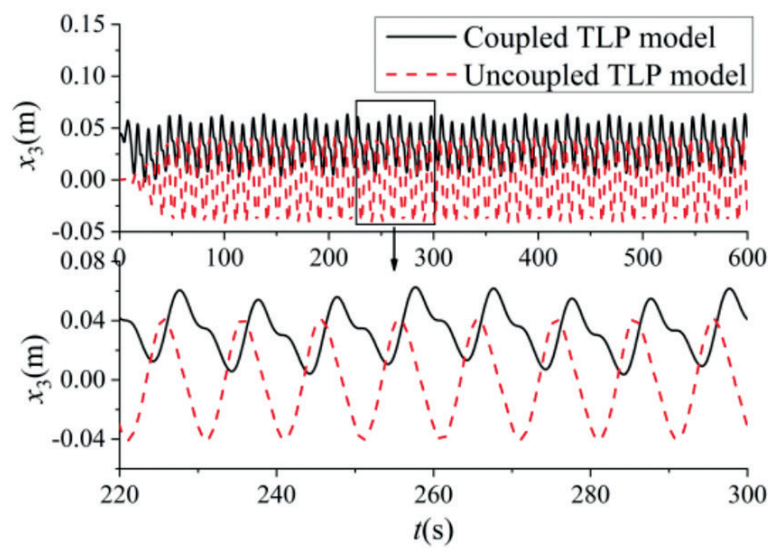

c) Heave motion

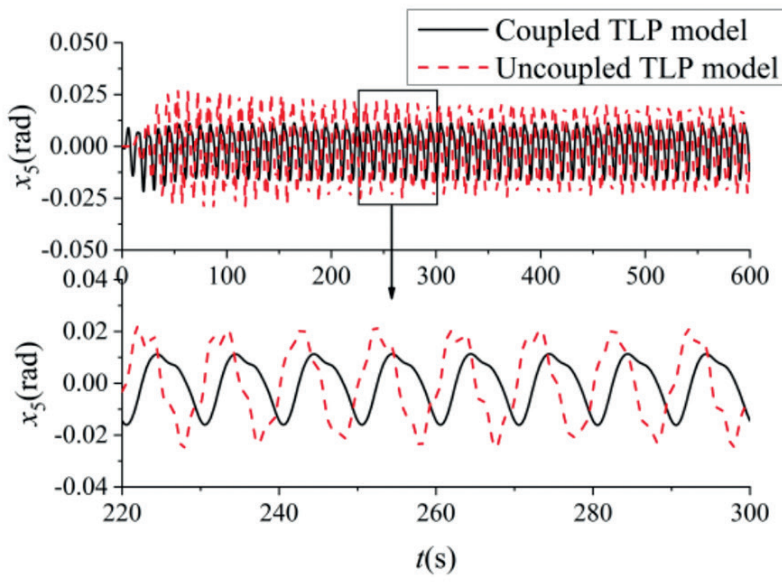

e) Pitch motion

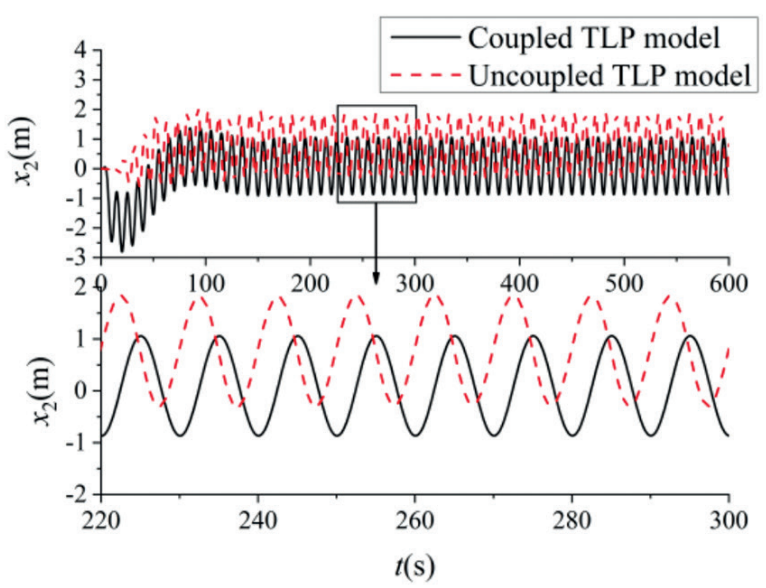

b) Sway motion

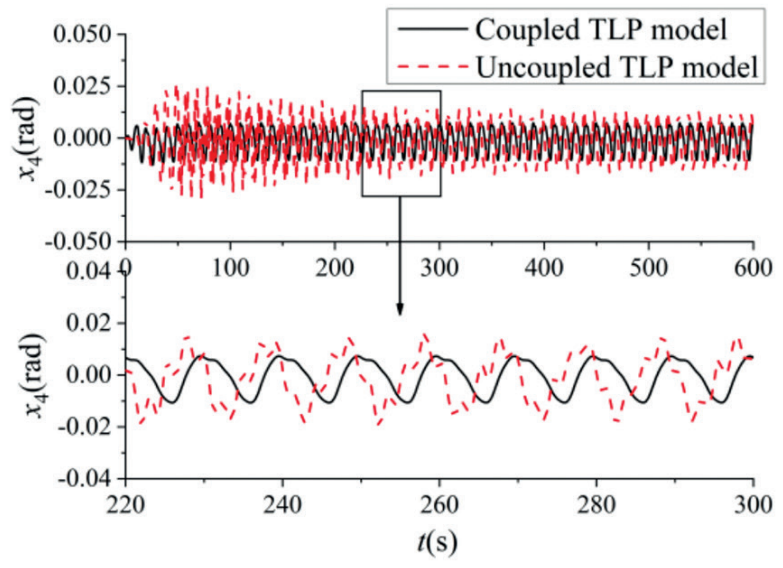

d) Roll motion

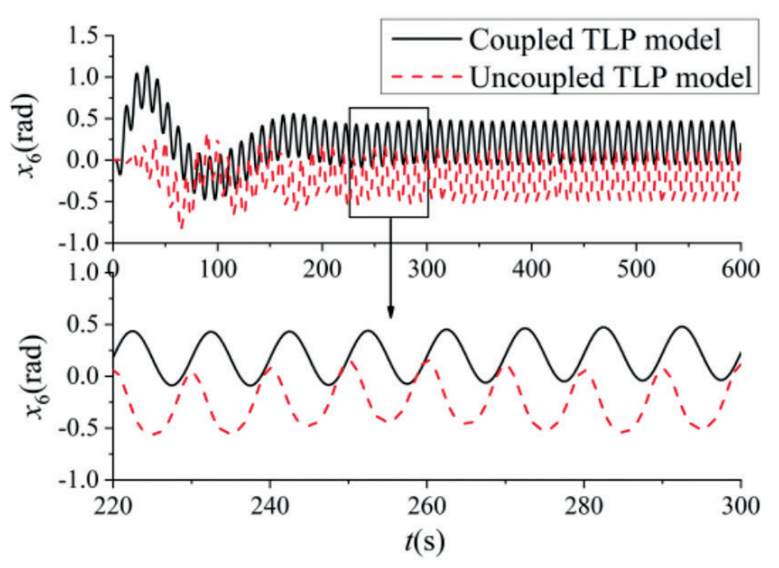

f) Yaw motion

Fig. 13. Comparison of motion responses of TLP under different calculation methods 
Tab. 4. Statistical table of motion responses of TLP

\begin{tabular}{|c|c|c|c|c|c|c|}
\hline & \multicolumn{2}{|c|}{ Coupled TLP model } & \multicolumn{2}{|c|}{ Uncoupled TLP model } & \multicolumn{2}{|c|}{ Relative errors } \\
\hline & Amplitude (m) & Freqency (rad/s) & Amplitude (m) & Freqency (rad/s) & Amplitude (\%) & Freqency (\%) \\
\hline Surge & 0.5302 & 0.637 & 0.5726 & 0.634 & 7.997 & 0.591 \\
\hline Sway & 0.91924 & 0.638 & 0.9821 & 0.634 & 6.838 & 0.689 \\
\hline \multirow[t]{2}{*}{ Heave } & 0.0313 & 0.599 & 0.0357 & 0.634 & 14.863 & 5.654 \\
\hline & Amplitude (rad) & Freqency (rad/s) & Amplitude (rad) & Freqency (rad/s) & Amplitude (\%) & Freqency (\%) \\
\hline Roll & 0.0086 & 0.599 & 0.0109 & 0.634 & 26.744 & 5.654 \\
\hline Pitch & 0.0136 & 0.598 & 0.0178 & 0.634 & 30.882 & 5.876 \\
\hline Yaw & 0.2439 & 0.637 & 0.2623 & 0.634 & 7.544 & 0.591 \\
\hline
\end{tabular}

is slightly smaller than that of the uncoupled TLP model. Among them, the relative errors of amplitude and frequency of surge, sway, and yaw motions of the two calculation models are relatively small; the average relative errors of amplitude and frequency are about $7.46 \%$ and $0.591 \%$, respectively. The relative errors of amplitude and frequency of roll, pitch, and heave motions are large, and the average relative errors of amplitude and frequency are about $24.16 \%$ and $5.728 \%$, respectively. The vibration of the tether in the uncoupled model is linear, and the vibration of the coupled model is non-linear. The influence of the tethers on the movements of the TLP is mainly reflected in the movement of the platform on the vertical plane, so the results of the platform motions on the vertical plane (heave, pitch, and roll) calculated by the two methods are quite different, while the results of the platform motions on the horizontal plane (surge, sway, and yaw) are less different.

Combined with the motion response results of TLP under the regular waves, the most obvious distinguishing feature of these two models is the platform's motion in the vertical plane (roll, pitch, and heave). Compared with the coupled TLP model, the uncoupled TLP model has higher oscillation frequencies and amplitudes. The main reason is that the mass of the tether is not taken into account in the uncoupled TLP model, and the tether is simplified as a spring structure with stiffness, ignoring the coupled effect of axial and transverse vibrations of the tethers. The consistency of the coupled and uncoupled TLP models is also noteworthy and demonstrates that the simpler system (the uncoupled TLP models) can be used to predict the surge/sway and sway motions. However, when calculating and studying the heave and roll/pitch motions of TLP or the motions of the tethers, the coupled TLP model is more suitable. In addition, the coupled TLP model considers both the coupled effect of the tether vibration and the coupled effect of platform motions. In contrast, the uncoupled TLP model only considers the coupled effect of platform motions, which also affects the movement of the platform in the vertical plane. To sum up, compared with the uncoupled TLP model, the coupled TLP model has the characteristics of higher precision of calculation results and wider application range.

\section{THE EFFECTS OF SECOND-ORDER IRREGULAR WAVE ON THE TETHER}

To study the influence of second-order wave forces acting on tether motion using the coupled TLP model, the motion responses of four design schemes are calculated in Table 5.

Tab. 5. Design scheme of the irregular waves

\begin{tabular}{|c|c|c|c|c|}
\hline & $\begin{array}{c}\text { First-order } \\
\text { wave force }\end{array}$ & $\begin{array}{c}\text { Mean drift } \\
\text { force }\end{array}$ & $\begin{array}{c}\text { Second- } \\
\text { order } \\
\text { differential } \\
\text { frequency } \\
\text { force }\end{array}$ & $\begin{array}{c}\text { Second-order } \\
\text { sum- } \\
\text { frequency } \\
\text { force }\end{array}$ \\
\hline case1 & $\checkmark$ & $\times$ & $\times$ & $\times$ \\
\hline case2 & $\checkmark$ & $\checkmark$ & $\times$ & $\times$ \\
\hline case3 & $\checkmark$ & $\times$ & $\checkmark$ & $\times$ \\
\hline case4 & $\checkmark$ & $\times$ & $\times$ & $\checkmark$ \\
\hline
\end{tabular}

Fig. 14 shows the comparative results of the surge motion responses of TLP under the second-order wave forces. As shown in Table 5, casel only considers the influence of firstorder wave force, case 2 considers both first-order wave force and mean drift force, case 3 considers both first-order wave force and second-order differential frequency force, and case 4 considers both first-order wave force and second-order sumfrequency force. As shown in Fig. 14a, the four design schemes have similar motion trajectories and different platform offset distances. Since the wave direction angle is $0^{\circ}$, the offset of the transverse vibration can be regarded as the offset of the platform.

The offsets of the four design schemes are calculated in Fig. 14b. Among them, the offsets of case 2 and case 3 are obviously large, while case 4 and case1 have little change, and their values are relatively close. It is verified that both the mean drift force and the second-order differential frequency force will cause low-frequency slow drift motion of the platform, resulting in the increase of the horizontal offset of the platform. Meanwhile, the second-order sum-frequency forces have little influence on it.

Filtering is performed on the history curve of the transverse vibration in Fig. 14a, and the filtering threshold is set as $0.157 \mathrm{rad} / \mathrm{s} \mathrm{(40} \mathrm{s)} \mathrm{to} \mathrm{obtain} \mathrm{the} \mathrm{high-frequency} \mathrm{curve} \mathrm{and} \mathrm{low-}$ frequency curve of the surge motion as shown in Fig. 14-d and 


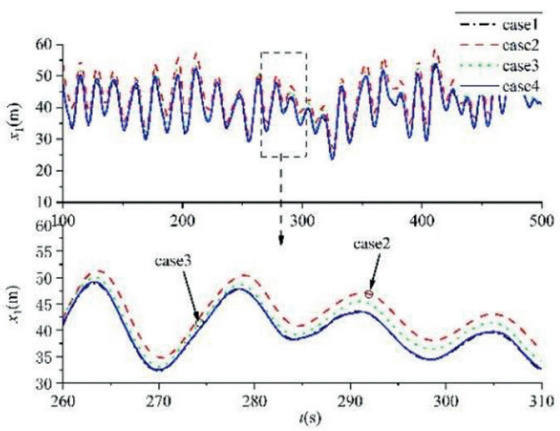

a) Time-history curve of surge motion

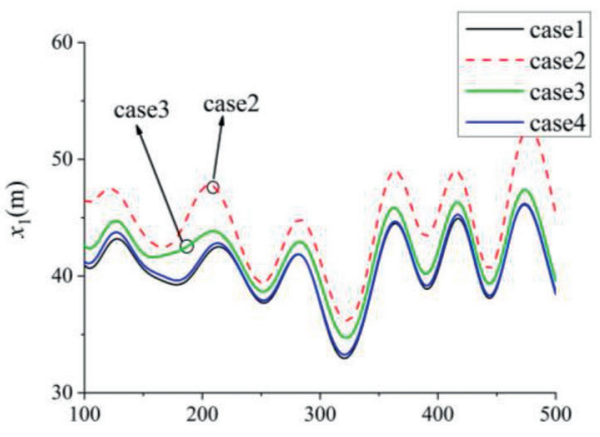

c) Low-frequency curve of surge motion

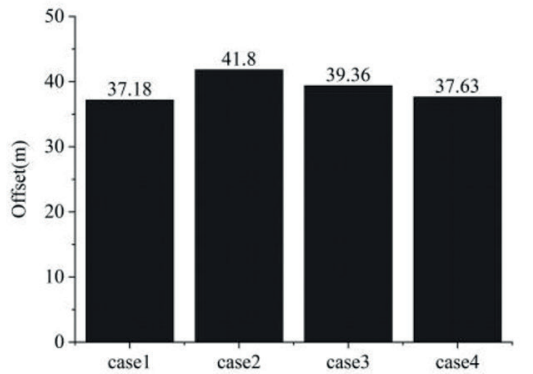

b) The offset of the platform

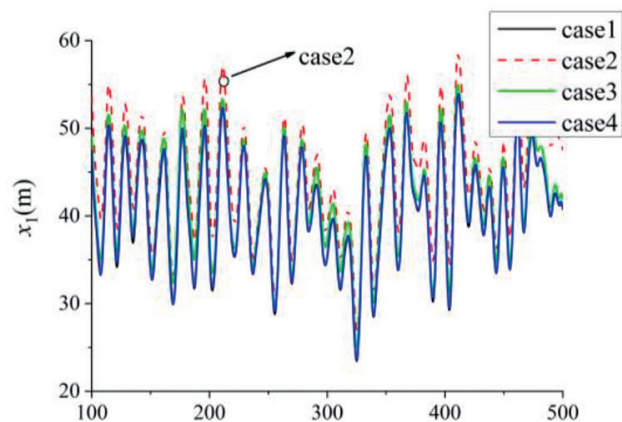

d) High-frequency curve of surge motion

Fig. 14. Comparison results of surge motion responses of TLP

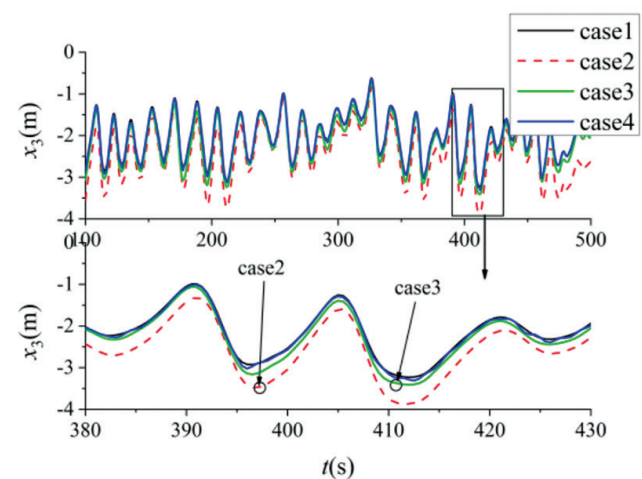

a) Time-history curve of heave motion

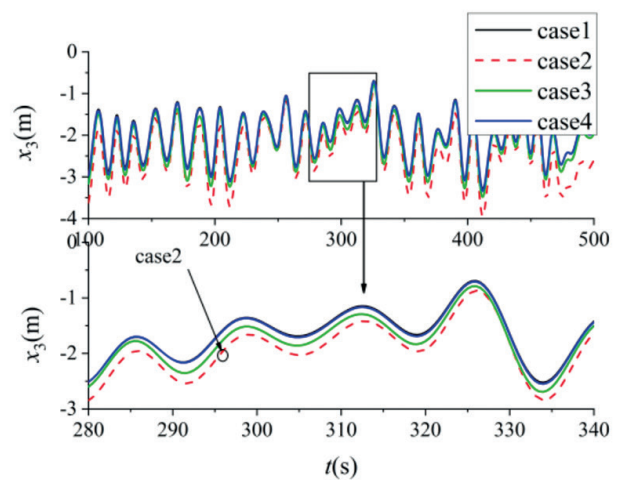

d) Low-frequency curve of heave motion

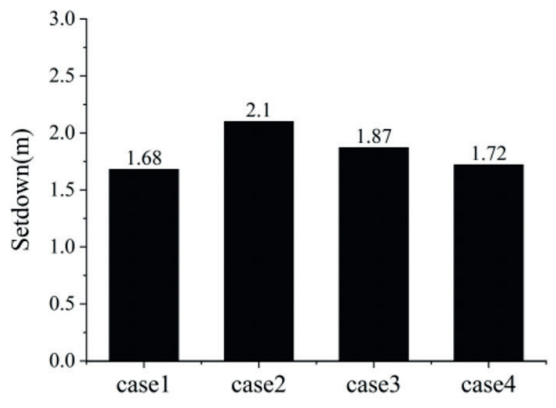

b) Setdown of the platform

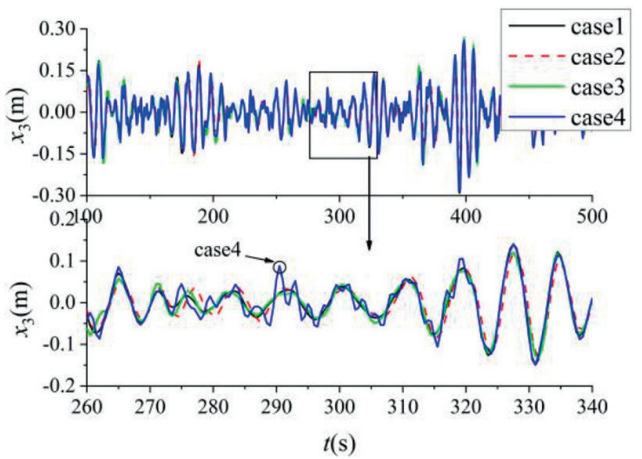

e) High-frequency curve of heave motion 
Fig. 14e. The low-frequency curve shows that both the mean offset force (case2) and the second-order sum-frequency force (case3) will cause the surge motion of the platform to occur in the long-period low-frequency floating motion, while the second-order high-frequency force (case4) has little effect on the surge motion of the platform.

Fig. 15 shows the heave motion responses of TLP under the second-order wave forces. The setdown of the platform has occurred in different degrees under the four different design schemes by observing Fig. 15b. The setdown of case2 (mean drift force) and case 3 (second-order differential frequency force) are large, similar to the platform's offset.

The time-history curve of the axial vibration in Fig. 15a is filtered, and the filtering threshold is 0.628 (10 s). By observing the local magnification of the low-frequency curve of axial vibration, there is no obvious change in the amplitude and vibration frequency of the tether, indicating that the secondorder wave force is not directly related to the setdown of the platform. The setdown of the platform is mainly caused by the coupled effect of surge/pitch motion and heave motion.

By observing the local magnification of the high-frequency curve of the axial vibration, the time history curve of case 4 (second-order sum-frequency force) moves at high frequency in the local time period, which shows that the second-order sum-frequency force will cause the high-frequency motion of the platform.

Table 6. The influence of wave force on platform motions

\begin{tabular}{|c|c|c|c|c|}
\hline & $\begin{array}{c}\text { First-order } \\
\text { wave force }\end{array}$ & $\begin{array}{c}\text { Mean drift } \\
\text { force }\end{array}$ & $\begin{array}{c}\text { Second- } \\
\text { order } \\
\text { differential } \\
\text { frequency } \\
\text { force }\end{array}$ & $\begin{array}{c}\text { Second-order } \\
\text { sum- } \\
\text { frequency } \\
\text { force }\end{array}$ \\
\hline $\begin{array}{c}\text { Surge } \\
\text { motion }\end{array}$ & $\begin{array}{c}\text { Wave } \\
\text { frequency } \\
\text { motion }\end{array}$ & Offset & Offset & $/$ \\
\hline $\begin{array}{c}\text { Heave } \\
\text { motion }\end{array}$ & $\begin{array}{c}\text { Wave } \\
\text { frequency } \\
\text { motion }\end{array}$ & Setdown & Setdown & $\begin{array}{c}\text { High- } \\
\text { frequency } \\
\text { motion }\end{array}$ \\
\hline
\end{tabular}

"/" represents wave forces have no influence on platform motions.

To sum up, Table 6 illustrates the influence of wave forces on platform motions. Both the mean wave drift force and the second-order differential frequency force will cause the offset of the platform. Additionally, the axial vibration will also produce the setdown phenomenon due to the influence of the platform motion coupled effect. The second-order drift force causes the axial vibration to move at a high frequency.

\section{CONCLUSION}

This paper has established two TLP models: the uncoupled TLP model and the coupled TLP model. In the uncoupled TLP model, the effects of horizontal offset and vertical setdown are considered in the restoring force coefficient matrix. In the coupled TLP model, the finite difference method is used to solve the non-linear tether vibration equation numerically.

By comparing the results of the TLP motion responses calculated by the two models, the conclusions are as follows: the average error between the results and the coupled TLP model is less than $10 \%$ when the platform's surge, sway, and yaw motions are calculated by the uncoupled TLP model. However, the model is simple and easy to implement. When studying the heave, roll, and pitch motions of TLP, the average error between the uncoupled TLP model and the coupled TLP model is greater than $15 \%$, and the coupled TLP model is more suitable.

Under the action of second-order wave force:

a) the second-order differential frequency force and the average drift force cause the horizontal offset of the platform.

b) The second-order sum-frequency forces have little influence on the movement in the horizontal plane but produce highfrequency movement in the vertical plane.

c) The average drift force and the second-order difference frequency force make the platform drift slowly.

This work was financially supported by National Natural Science Foundation of China (No. 52075469).

\section{APPENDIX A: HYDRODYNAMIC COEFFICIENT OF THE PLATFORM}

BEM (boundary element method) determines the hydrodynamic coefficient of the platform under the action of waves. Since the platform is a slender structure, the effect of wave viscosity on the slender structure cannot be ignored. Then, the drag force on the floating structure is determined by the Morison equation. Therefore, the hydrodynamic coefficient of the platform is calculated by the methods of BEM and the Morison equation.

\section{Added mass and memory function}

The added mass and memory function can be determined by calculating the radiation potential of the platform. The integral equation of the radiation potential is calculated by using the boundary element method. The expression $[21,22]$ is shown as follows:

$$
\begin{gathered}
2 \pi \Phi_{k}(p, t)+\iint_{s_{b}}\left[\begin{array}{c}
\Phi_{k}(q, t) \frac{\partial}{\partial n_{q}}\left(\frac{1}{r}-\frac{1}{r^{\prime}}\right) \\
-\left(\frac{1}{r}-\frac{1}{r^{\prime}}\right) \frac{\partial \Phi_{k}(q, t)}{\partial n_{q}}
\end{array}\right] d S_{q}= \\
\int_{0}^{t} d \tau \iint_{s_{b}}\left(\begin{array}{l}
\widetilde{G}(p, t ; q \tau) \frac{\partial \Phi_{k}(q, t)}{\partial n_{q}} \\
-\Phi_{k}(q, t) \frac{\partial \widetilde{G(p, t ; q, \tau)}}{\partial n_{q}}
\end{array}\right) d S_{q}
\end{gathered}
$$

where $\Phi_{k}$ is the velocity potential that needs to be evaluated. The coordinates of field point $p$ and source point $q$ are $(x, y, z)$ and $(\xi, \eta, \zeta)$, respectively, with both lying in the water $(z \leq 0)$. $R=\sqrt{(x-\xi)^{2}+(y-\eta)^{2}}, r=\sqrt{R^{2}+(z-\zeta)^{2}}$, and $r^{\prime}=\sqrt{R^{2}+(z-\zeta)^{2}}$ represent the horizontal or the three-dimensional distance between field point $p$ and source point $q$ or the ' $q$ s image about the still water surface. $s_{b}$ is the wet surface of the floating structure. $n_{q}$ is the unit normal vector of any source point on the wet surface. $\widetilde{G}(p, t ; q, \tau)$ is the memory term of the corresponding time-domain Green function. Its expression 
[23] is:

$$
\widetilde{G}(p, q ; t)=2 \int_{0}^{\infty} d k \sqrt{g k} \sin (\sqrt{g k t}) e^{k(z+\zeta)} J_{0}(k R)
$$

where $J_{0}$ is the Bessel function of the first kind with order 0 .

The calculation method of the Green function is based on Clement [8]. The memory of the Green function is transformed into a fourth-order ordinary differential equation, and then the Runge-Kutta method is adopted to calculate the differential equation.

According to the method of Fual [9], Eq. (57)is decomposed into instantaneous part and memory part. The expressions of them are:

$$
\begin{aligned}
& \psi_{k}(p)+\frac{1}{2 \pi} \iint_{S_{b}} \psi_{k}(q) \frac{\partial}{\partial n_{q}}\left(\frac{1}{r}-\frac{1}{r^{\prime}}\right) d S_{q} \\
& =\frac{1}{2 \pi} \iint_{s_{b}}\left(\frac{1}{r}-\frac{1}{r^{\prime}}\right) n_{k} d S_{q} \\
& \chi_{k}(p, t)+\frac{1}{2 \pi} \iint_{s_{b}} \chi_{k}(q, t) \frac{\partial}{\partial n_{q}}\left(\frac{1}{r}-\frac{1}{r^{\prime}}\right) d S_{q} \\
& =\frac{1}{2 \pi} \iint_{s_{b}} n_{k}(q) \tilde{G}(p, t ; q, 0) d S_{q} \\
& -\frac{1}{2 \pi} \iint_{s_{b}} \psi_{k}(q) \frac{\partial}{\partial n_{q}} \tilde{G}(p, t ; q, 0) d S_{q} \\
& -\frac{1}{2 \pi} \int_{0}^{t} d \tau \iint_{s_{b}} \chi_{k}(q, \tau) \frac{\partial \tilde{G}(p, t ; q, \tau)}{\partial n_{q}} d S_{q}
\end{aligned}
$$

Eqs. (59)and (60) are numerically discrete. A series of quadrilateral grids use the wet surface of the $3 \mathrm{D}$ object to approximate. In each quadrilateral grid, the velocity potential is taken as a constant, and then the algebraic equation sets of instantaneous part and memory part are:

$$
\begin{array}{cc}
\sum_{j=1}^{M} A_{i j}\left(\psi_{k}\right)_{j}=B_{i} & i=1,2, \ldots, M \\
\sum_{j=1}^{M} A_{i j}\left[\chi_{k}\left(t_{N}\right)\right]_{j}=B\left(t_{N}\right)_{i} & i=1,2, \ldots, M
\end{array}
$$

where $M$ is the number of quadrilateral elements; $N$ is the current time steps; $\left(\psi_{k}\right)_{j}$ is the instantaneous part of the $j$ element; $\left[\chi_{k}\left(t_{N}\right)\right]_{j}$ is the memory part of the $\mathrm{j}$ element in the time of $t_{N} ; A_{i j}, B_{i}$, and $B\left(t_{N}\right)_{i}$ are the coefficient matrixes, and the expressions are: $\left\{\begin{array}{l}\iint_{S_{\jmath}} \frac{\partial}{\partial n_{\jmath}}\left(\frac{1}{r_{\iota \jmath}}-\frac{1}{r_{\iota \jmath}}\right) d S, \quad i \neq j \\ 2 \pi-\iint_{s_{\jmath}} \frac{\partial}{\partial n_{\jmath}}\left(\frac{1}{r_{\iota \jmath}}\right) d S, i=j\end{array}\right.$

$$
\begin{gathered}
B_{\imath}=\sum_{J=1}^{M} \iint_{s_{J}}\left(\frac{1}{r_{\iota \jmath}}-\frac{1}{r_{\iota \jmath}}\right)\left(n_{k}\right)_{J} d S \\
B\left(t_{N}\right)_{\iota}=\sum_{J=1}^{M} \iint_{S_{J}}\left[n_{k}\right]_{J} \tilde{G}\left(p_{l}, q_{J}, t_{N}\right) d S \\
-\sum_{J=1}^{M} \iint_{S_{J}}\left[\psi_{k}\right]_{J} \frac{\partial}{\partial n_{J}} \tilde{G}\left(p_{l}, q_{J}, t_{N}\right) d S \\
-\Delta t \sum_{n=1}^{N} \sum_{J=1}^{M} \iint_{S_{J}}\left[\chi_{k}\left(q_{J}, t_{n}\right)\right]_{J} \frac{\partial}{\partial n_{J}} \tilde{G}\left(p_{l}, q_{J}, t_{N}-t_{n}\right) d S
\end{gathered}
$$

By the calculation of algebraic equation sets, the integral equations of added mass and memory function are:

$$
\begin{gathered}
\mu_{i k}=\rho \iint_{s_{b}} \psi_{k}(q) n_{j} d S_{q} \\
\bar{K}_{j k}(t)=\rho \iint_{s_{b}} \frac{\partial}{\partial t} \chi_{k}(q, t) d S_{q} \\
A_{j k}(\omega)=\mu_{i k}-\frac{1}{\omega} \int_{0}^{t} K_{j k}(\tau) \sin (\omega \tau) d \tau \\
B_{j k}(\omega)=\int_{0}^{t} K_{j k}(\tau) \cos (\omega \tau) d \tau
\end{gathered}
$$

where $\bar{K}_{j k}(t)$ is the memory function; $A_{j k}(\omega)$ is the added mass; $B_{j k}(\omega)$ is the damping coefficient.

\section{The damping matrix}

The damping matrix assumption is related to the mass matrix and the restoring force matrix, which can be expressed as follows:

$$
\Phi^{T}[K] \Phi=\left[2 \zeta_{i} \omega_{i} m_{i}\right]
$$

where $\Phi$ is the shape matrix $\omega_{i}$ is the structure natural frequency $K$ is the damping matrix $m_{i}$ is the modal mass $m_{i}=\Phi^{T}[M] \Phi, \zeta$ is the dimensionless damping ratio of each mode, generally 0.05 .

The solution of $\Phi$ and $\omega_{i}$ is presented below.

For the undamped vibration:

$$
M \ddot{Y}+K Y=0
$$

The characteristic determinant is:

$$
|T-\lambda M|=0
$$

The characteristic vector $\left[\Phi_{1}, \Phi_{2} \ldots \Phi_{\mathrm{N}}\right]$ and characteristic value $\left[\begin{array}{llll}\lambda_{1} & & & \\ & \lambda_{2} & & \\ & & \ddots & \\ & & \lambda_{n}\end{array}\right]$ are obtained,

$$
\lambda_{i}=\omega_{i}^{2} \quad \omega_{i}=\sqrt{\lambda_{i}}
$$

The modal mass is

$$
\left[\begin{array}{llll}
m_{1} & & & \\
& m_{2} & \\
& & \ddots & \\
& & m_{n}
\end{array}\right]=[\Phi]^{T}[M][\Phi]
$$

The $K$ matrix can be obtained by solving the following matrix equation:

$$
\Phi^{T}[c] \Phi=2 \xi_{i}\left[\begin{array}{llll}
\sqrt{\lambda}_{1} & & & \\
& \sqrt{\lambda}_{2} & & \\
& & \ddots & \\
& & \sqrt{\lambda}_{n}
\end{array}\right]\left[\begin{array}{llll}
m_{1} & & \\
& & m_{2} & \\
& & \ddots & \\
& & & m_{n}
\end{array}\right]
$$

\section{WAVE FORCE}

\section{Froude-Krylov force}

Froude-Krylov force is caused by the incident potential, and its expression is:

where $\Delta t$ is the time step; $t_{N}=N \Delta t ; t_{n}=n \Delta t$. 


$$
F_{j I}(t)=\iint_{s_{b}} p(q, t) n_{j} d S_{q}
$$

where $F_{j I}$ is the Froude-Krylov force in the $j(j=1,2, \ldots, 6)$ mode of motions. $n_{j}$ is the unit surface normal vector.

The expression [10] of the dynamic pressure acting on the floating structure by the incident waves is:

$$
p(p, t)=\int_{-\infty}^{+\infty} \hat{p}(p, t-\tau) \zeta_{0}(\tau) d \tau
$$

where $\hat{p}(p, t)$ is the impulse response function of pressure. The expression is:

$$
\hat{p}(p, t)=\frac{\rho g}{\pi} \operatorname{Re}\left\{\int_{0}^{\infty} e^{k(z-i \alpha)} e^{i \omega t} d \omega\right\}
$$

When the incident wave is a regular wave per unit wavelength, $\zeta_{0}(t)=e^{i \omega t}$.

\section{Diffraction force}

The diffraction force is mainly caused by the diffraction potential, and its expression is:

$$
\begin{aligned}
& 2 \pi \widehat{\Phi}_{7}(p, t)+\iint_{s_{b}} \widehat{\Phi}_{7}(q, t) \frac{\partial}{\partial n_{q}}\left(\frac{1}{r}-\frac{1}{r^{\prime}}\right) d S_{q} \\
& =-\iint_{s_{b}}\left(\frac{1}{r}-\frac{1}{r^{\prime}}\right)[\overrightarrow{\boldsymbol{n}} \cdot \widehat{K}(q, t)] d S_{q} \\
& \quad-\int_{0}^{t} d \tau \iint_{s_{b}}\left\{\begin{array}{l}
\tilde{G}(p, t ; q, \tau)[\overrightarrow{\boldsymbol{n}} \cdot \widehat{K}(q, t)] \\
+\widehat{\Phi}_{7}(q, \tau) \frac{\partial \tilde{G}(p, t, q, \tau)}{\partial n_{q}}
\end{array}\right\} d S_{q}
\end{aligned}
$$

where the impulse response function $\widehat{K}(p, t)$ is:

$$
\widehat{K}(p, t)=\frac{1}{\pi} \operatorname{Re}\left\{\left[\begin{array}{l}
\overrightarrow{\boldsymbol{i}} \cos \beta \\
\overrightarrow{\boldsymbol{j}} \sin \beta \\
\overrightarrow{\boldsymbol{k}} i
\end{array}\right] \int_{0}^{\infty} e^{k(z-\iota \alpha)} e^{i \omega t} d \omega\right\}
$$

The calculation method of diffraction potential is the same as that of radiation potential. By solving the integral equation of the diffraction potential, the expression of diffraction force is:

$$
F_{j 7}=\int_{-\infty}^{\infty} \zeta_{0}(\tau) d \tau\left[-\rho \iint_{s_{b}} \frac{\partial}{\partial t} \widehat{\Phi}_{7}(q, t-\tau) n_{j} d S_{q}\right]
$$

\section{Drag force}

For slender columns, the influence of the drag force on the platform can be calculated by the Morison equation, and the drag force per unit length of column is:

$$
f_{D}=\frac{1}{2} \rho C_{D} D\left(w_{x}-\dot{x}\right)\left|\left(w_{x}-\dot{x}\right)\right|
$$

where $D$ is the diameter of the column; $C D$ is the coefficient of drag force; $w_{x}$ is the horizontal component of the velocity of the fluid particle; $\dot{x}$ is the velocity of a floating structure.

The determination method of drag force coefficient [11] is:

$$
C_{D}= \begin{cases}60.566-5.93 R e & \operatorname{Re} \leq 10 \\ 1.25 & 10<\operatorname{Re} \leq 5 \times 10^{5} \\ 0.7 & \operatorname{Re}>5 \times 10^{5}\end{cases}
$$

where: Re is the Reynolds number, that is, $R e=U D / v$. $U$ is the relative velocity of the fluid. $D$ is characteristic length. In this paper, is the diameter of the column. $v$ is the coefficient of kinematic viscosity, which is $1.346 \mathrm{eE}-6 \mathrm{~m}^{2} / \mathrm{s}$ when the temperature of seawater is $10^{\circ} \mathrm{C}$.

The velocity of a fluid particle is calculated by the Airy linear wave theory. The velocity of water particles (x-direction) is:

$$
W_{x^{\prime}}=\frac{\pi W_{H}}{W_{T}} \frac{\sinh (k x)}{\sinh (k d)} \sin (k z-\omega t)
$$

The velocity of water particles (y-direction) is:

$$
W_{y^{\prime}}=0
$$

The velocity of water particles (z-direction) is:

$$
W_{z^{\prime}}=\frac{\pi W_{H}}{W_{T}} \frac{\cosh (k x)}{\sinh (k d)} \cos (k z-\omega t)
$$

where $W_{H}$ is the wave height; $W_{T}$ is the wave period; $k$ is the wave number; $\omega$ is the wave frequency; $d$ is the water depth.

According to the method of the coordinate transformation, it transforms the particle velocity in the wave coordinate system $O^{\prime} X^{\prime} Y^{\prime} Z^{\prime}$ to the static coordinate system OXYZ:

$$
\left\{\begin{array}{l}
W_{x}=W_{x^{\prime}} \cos \alpha \\
W_{y}=W_{y^{\prime}} \cos \alpha \\
W_{z}=W_{z^{\prime}}
\end{array}\right.
$$

$\alpha$ is the angle between the coordinate system $O^{\prime} X^{\prime} Y^{\prime} Z^{\prime}$ and the static coordinate system OXYZ.

The relative velocity between water particle and floating structure in the static coordinate system OXYZ is:

$$
\begin{gathered}
\vec{V}=\left(\dot{x}_{1} \vec{i}+\dot{x}_{2} \vec{J}+\dot{x}_{3} \vec{k}\right)+\vec{\omega} \times \vec{r} \\
\vec{r}=x_{1} \vec{i}+x_{2} \vec{J}+x_{3} \vec{k}
\end{gathered}
$$

where: $\vec{\omega} \times \vec{r}=\left|\begin{array}{ccc}\vec{i} & \vec{J} & \vec{k} \\ \dot{x}_{4} & \dot{x}_{5} & \dot{x}_{6} \\ x_{1} & x_{2} & x_{3}\end{array}\right|$, and substitute it into Eq. (88). Then the relative velocity is:

$$
\begin{aligned}
& \vec{w}_{r}=w_{r x} \vec{i}+w_{r y} \vec{J}+w_{r z} \vec{k}=\left[w_{x}-\left(\dot{x}_{5} x_{3}-\dot{x}_{6} x_{2}+\dot{x}_{1}\right)\right] \vec{i}+ \\
& {\left[w_{y}-\left(-\dot{x}_{4} x_{3}-\dot{x}_{6} x_{1}+\dot{x}_{2}\right)\right] \vec{J}+\left[w_{z}-\left(\dot{x}_{4} x_{2}-\dot{x}_{5} x_{1}+\dot{x}_{3}\right)\right] \vec{k}}
\end{aligned}
$$

The wet surface of the platform is separated into a series of differential rings, and the centres of the circles are taken as discrete points. Based on the Morison equation, the drag force calculating formula in the case of the $i$ th discrete element is:

$$
\left[\begin{array}{l}
F_{D x i} \\
F_{D y i} \\
F_{D z i}
\end{array}\right]=\frac{1}{2} \rho C_{D} D\left(\begin{array}{l}
w_{r x i} \\
w_{r y i} \\
w_{r z i}
\end{array}\right)\left|\begin{array}{l}
w_{r x i} \\
w_{r y i} \\
w_{r z i}
\end{array}\right|
$$

The calculation formula of the drag forces of the platform in waves is:

$$
F_{D x} \quad \sum_{i=1}^{N} F_{D x i}
$$




$$
\left\{\begin{array}{l}
F_{D y}=\left\{\begin{array}{l}
\sum_{i=1}^{N} F_{D y i} \\
F_{D z}^{N}
\end{array} \sum_{i=1}^{N} F_{D z i}\right.
\end{array}\right.
$$

where $N$ is the number of discrete elements on the platform.

The calculation formula of the drag moments of the platform in waves is:

$$
\left\{\begin{array}{l}
M_{D x}=F_{D z} y-F_{D y} z \\
M_{D y}=F_{D x} z-F_{D z} x \\
M_{D z}=F_{D y} x-F_{D x} y
\end{array}\right.
$$

When the relative velocities are calculated, the velocities of the platform are required. Therefore, the formulas of wave drag forces are linked with the differential motion equations of the floating structure, and the time iteration of the differential equations is carried out. Then the drag forces of waves on the platform can be calculated.

\section{Second-order wave forces}

The wave force on the platform includes not only the firstorder wave force calculated above but also the second-order wave force. The second-order wave forces consist mainly of the mean drift force, the sum-frequency wave force, and the differential frequency wave force [20].

There are two methods of calculating the mean drift force, including the near-field method and the far-field method. The near-field method is based on the direct solution of the waterline surface and object area components to obtain the average drift force for the six degrees of freedom of the platform. The far-field method is achieved mainly through the law of conservation of energy. In contrast, the far-field method can only result in the calculation of the platform's component in the horizontal plane. The near-field and far-field formulations of the mean drift force are given separately $[21,22]$.

The near-field equation for mean drift force is:

$$
\begin{aligned}
& F_{d x}=-\frac{\rho}{2}\left\{\iint_{s_{H}}\left[\phi_{1} \frac{\partial}{\partial n}\left(\frac{\partial \phi_{1}}{\partial x}\right)-\frac{\partial \phi_{1}}{\partial x} \cdot \frac{\partial \phi_{1}}{\partial n}\right] d s\right\}_{m} \\
& F_{d y}=-\frac{\rho}{2}\left\{\iint_{s_{H}}\left[\phi_{1} \frac{\partial}{\partial n}\left(\frac{\partial \phi_{1}}{\partial y}\right)-\frac{\partial \phi_{1}}{\partial y} \cdot \frac{\partial \phi_{1}}{\partial n}\right] d s\right\}_{m}
\end{aligned}
$$

while the far-field equation for mean drift force is:

$$
\begin{gathered}
F_{d x}=-\frac{\rho}{2 g}\left\{\int_{l_{0}}\left(\frac{\partial \phi_{1}}{\partial t}\right)^{2} n_{x} d l\right\}_{m}- \\
\rho\left\{\iint_{C_{0}}\left[\frac{\partial \phi_{1}}{\partial x} \cdot \frac{\partial \phi_{1}}{\partial n}-\frac{1}{2}\left(\nabla \phi_{1}\right)^{2} n_{x}\right] d s\right\}_{m} \\
F_{d y}=-\frac{\rho}{2 g}\left\{\int_{l_{0}}\left(\frac{\partial \phi_{1}}{\partial t}\right)^{2} n_{y} d l\right\}_{m}- \\
\rho\left\{\iint_{C_{0}}\left[\frac{\partial \phi_{1}}{\partial y} \cdot \frac{\partial \phi_{1}}{\partial n}-\frac{1}{2}\left(\nabla \phi_{1}\right)^{2} n_{y}\right] d s\right\}_{m}
\end{gathered}
$$

As the near-field formulation of the mean drift force can calculate the motion of the platform in six degrees of freedom, the far-field formulation can only calculate the surge, sway, and yaw motions, this paper focuses on the effect of the mean drift force calculated by the near-field method on the motion response of the TLP.

The expression for the second-order force [21, 22] can be expressed as:

$$
F^{(2)}=\frac{1}{2} \rho g \int_{\Gamma 0} \eta^{(1)^{2}} n d \Gamma-\rho \iint_{S}\left(\phi_{t}^{(2)}+\frac{1}{2}\left(\nabla \phi^{(1)}\right)^{2}\right) n d S
$$

The above equation is bounded using second-order bypassing-radiation theory, and the standard form of the second-order force calculation equation:

$$
\begin{gathered}
F^{(2)}(t)=A_{1}^{2} f_{d}\left(\omega_{1}\right)+h\left\{A_{1}^{2} f_{+}^{(2)}\left(\omega_{1}, \omega_{1}\right) e^{-2 i \omega_{1} t}+\right. \\
A_{2}^{2} f_{+}^{(2)}\left(\omega_{2}, \omega_{2}\right) e^{-2 i \omega_{2} t}+2 A_{1} A_{2} f_{-}^{(2)}\left(\omega_{1}, \omega_{2}\right) e^{-2 i\left(\omega_{1}-\omega_{2}\right) t}+ \\
\left.2 A_{1} A_{2} f_{+}^{(2)}\left(\omega_{1}, \omega_{2}\right) e^{-2 i\left(\omega_{1}+\omega_{2}\right) t}\right\}+A_{2}^{2} f_{d}\left(\omega_{2}\right)
\end{gathered}
$$

where $f_{d}$ is the normalised mean drift force; $f_{+}^{(2)}$ is the secondorder transfer function (QTF) of the normalised sum-frequency force; $f_{-}^{(2)}$ is the second-order transfer function (QTF) of the normalised difference frequency force; $\omega_{1}$ and are the $\omega_{2}$ wave frequencies of the two incident waves; $A_{1}$ and $A_{2}$ are the wave amplitudes of the two incident waves respectively.

In this paper, the frequency domain solution of the secondorder force is calculated based on the HydroD module, including the average drift force $f_{d}$ and the second-order transfer function (QTF) of the sum-frequency force and differential-frequency force, respectively, and embedded in the coupled TLP model compiled in this paper to obtain the TLP motion responses under the influence of the second-order wave forces.

\section{REFERENCES}

1. Petrolio, 'American Petroleum Institute', Acta Petrolei Sinica, vol. 26, pp. 139-177, 1982.

2. J. R. Paulling and E. E. Horton, 'Analysis of the tension leg platform', Society of Petroleum Engineers Journal, vol. 11, no. 03, pp. 285-294, 1971.

3. J. Paulling, 'Time-domain simulation of semi-submersible platform motion with application to the tension leg platform', 1977.

4. D. C. Angelides, C. Chen, and S. A. Will, 'Dynamic response of tension leg platform, 1982.

5. S. Chandrasekaran and A. K. Jain, 'Dynamic behaviour of square and triangular offshore tension leg platforms under regular wave loads', Ocean Engineering, vol. 29, no. 3, pp. 279-313, 2002.

6. S. Chandrasekaran and A. K. Jain, 'Triangular configuration tension leg platform behaviour under random sea wave loads', Ocean Engineering, vol. 29, no. 15, pp. 1895-1928, 2002. 
7. T. Kawanishi, W. Katoh, and H. Furuta, 'Tension leg platform earthquake motion analysis, IEEE, 1987.

8. G. J. Zhai, D. Y. Tang, and H. F. Xiong, 'Numerical simulation of the dynamic behavior of deep-water semi-submersible platform under wind and waves', Advanced Materials Research, vol. 243-249, pp. 4733-4740, 2011.

9. S. Chandrasekaran, N. R. Chandak, and G. Anupam, 'Stability analysis of TLP tethers', Ocean Engineering, vol. 33, no. 3/4, pp. 471-482, 2006.

10. Y. M. Low, 'Frequency domain analysis of a tension leg platform with statistical linearization of the tendon restoring forces', Marine Structures, vol. 22, no. 3, pp. 480-503, 2009.

11. Senjanović, I. Hadžić, N. Tomić, and Marko, 'On the linear stiffness of tension leg platforms', Sustainable Maritime Transportation and Exploitation of Sea Resources / Enrico, Rizzuto; Carlos Guedes Soares, 2011.

12. K. Kozak, Z. Qian, and J. Wang, 'Static analysis of cabledriven manipulators with non-negligible cable mass', IEEE Press, 2006.

13. M. Jameel, D. O. Oyejobi, N. A. Siddiqui, and N. Sulong, 'Non-linear dynamic response of tension leg platform under environmental loads', KSCE Journal of Civil Engineering, vol. 21, no. 3, pp. 1-9, 2017.

14. A. K. Jain, 'Non-linear coupled response of offshore tension leg platforms to regular wave forces', Ocean Engineering, vol. 24, no. 7, pp. 577-592, 1997.

15. O. M. Faltinsen, Sea Loads on Ships and Offshore Structures, 1990.

16. P. C. Chatterjee, P. K. Das, and D. Faulkner, 'A hydrostructural analysis program for TLPS', Ocean Engineering, vol. 24, no. 4, pp. 313-334, 1997.

17. Y. M. Choi, B. W. Nam, S. Y. Hong, D. W. Jung, and H. J. Kim, 'Coupled motion analysis of a tension leg platform with a tender semi-submersible system', Ocean Engineering, vol. 156, no. MAY15, pp. 224-239, 2018.

18. Z. Ran, M. H. Kim, and W. Zheng, 'Coupled dynamic analysis of a moored spar in random waves and currents (time-domain versus frequency-domain analysis)', Journal of Offshore Mechanics \& Arctic Engineering, vol. 121, no. 3, p. $194,1999$.

19. T. Mazarakos and S. A. Mavrakos, 'Mean second-order wave drift forces contour of a floating structure concept for wind energy exploitation', in 4th International Conference on Renewable Energies Offshore (RENEW 2020), 2020.
20. S. G. Tan and W. D. Boom, 'The wave induced motions of a tension leg platform in deep water, Offshore Technology Conference, 1981.

21. E. L. Günther Clauss and C. Östergaard, Offshore Structures. Springer Berlin, 1992.

22. Chakrabarti, 'Handbook of offshore engineering', pp. 79-131, 2005.

23. J. Wang, L. Sun, Y. Wang, and S. Dai, 'A method based on LS-SVM to estimate time-domain Green function', Journal of Marine Science and Technology, no. 5, pp. 1-13, 2020.

\section{CONTACT WITH THE AUTHORS}

\author{
Jianwei Wang \\ e-mail:wangjianwei@ysu.edu.cn \\ Yanshan University \\ Hebei Street, 066004 Qinhuangdao \\ China
}

Yuankui Luo

e-mail:luoyuankui2020@163.com

Yanshan University

Hebei Street, 066004 Qinhuangdao

China

Rui Guo

e-mail:guorui@ysu.edu.cn

Yanshan University

Hebei Street, 066004 Qinhuangdao

China

Wei Cai

e-mail:caiwei@ysu.edu.cn

Yanshan University

Hebei Street, 066004 Qinhuangdao

China

\section{Jingyi Zhao}

e-mail: zjy@ysu.edu.cn

Yanshan University

Hebei Street, 066004 Qinhuangdao

China

Xiongshuai Luo

e-mail:1264600281@qq.com

Yanshan University

Hebei Street, 066004 Qinhuangdao China 